\title{
Solution of the KdV equation on the line with analytic initial potential
}

\author{
Andrey Melnikov \\ Drexel University, Philadelphia, USA
}

October 6, 2018

\begin{abstract}
We present a theory of Sturm-Liouville non-symmetric vessels, realizing an inverse scattering theory for the Sturm-Liouville operator with analytic potentials on the line. This construction is equivalent to the construction of a matrix spectral measure for the Sturm-Liouville operator, defined with an analytic potential on the line. Evolving such vessels we generate KdV vessels, realizing solutions of the $\mathrm{KdV}$ equation. As a consequence, we prove the following theorem:

Suppose that $q(x)$ is an analytic function on $\mathbb{R}$. There exists a KdV vessel, which exists on $\Omega \subseteq \mathbb{R}^{2}$. For each $x \in \mathbb{R}$ there exists $T_{x}>0$ such that $\{x\} \times\left[-T_{x}, T_{x}\right] \in \Omega$. The potential $q(x)$ is realized by the vessel for $t=0$.

Since we also show that if $q(x, t)$ is a solution of the $\mathrm{KdV}$ equation on $\mathbb{R} \times\left[0, t_{0}\right)$, then there exists a vessel, realizing it, the theory of vessels becomes a universal tool to study this problem. Finally, we notice that the idea of the proof applies to a similar existence of a solution for evolutionary NLS and Boussinesq equations, since both of these equations possess vessel constructions.
\end{abstract}

\section{Contents}

1 Introduction

2 Background on Krein space theory 5

3 Non-symmetric vessels 7

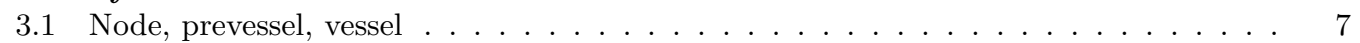

3.2 Standard construction of a prevessel . . . . . . . . . . . . . . . 11

3.3 Tau function of a prevessel . . . . . . . . . . . . . . . . . 14

3.4 Moments and their properties . . . . . . . . . . . . . . . 15

4 Sturm-Liouville vessels $\quad 16$

4.1 Construction of a realized function, possessing given Moments . . . . . . . . . . . . 17

4.2 Construction of a vessel, realizing a given analytic potential . . . . . . . . . . 22

\begin{tabular}{lll}
5 & $\mathrm{KdV}$ evolutionary vessels & 26 \\
\hline
\end{tabular}

6 Remarks $\quad 32$ 


\section{Introduction}

The Korteweg-de Vries (KdV) is the following nonlinear evolutionary Partial Differential Equation (PDE) for a function of two real variables $q(x, t)$ :

$$
q_{t}=-\frac{3}{2} q q_{x}+\frac{1}{4} q_{x x x}
$$

where $q_{t}, q_{x}$ denote the partial derivatives. The equation is named after Diederik Korteweg and Gustav de Vries who studied it in KdV95. Usually, one considers the initial value problem, which is defined as follows: find a solution $q(x, t)$ of (1), which additionally satisfies: $q(x, 0)=q(x)(x \in \mathbb{R})$, for a given function $q(x)$, defined on $\mathbb{R}$. A standard technique to solve KdV involves a more elementary equation, called Sturm Liouville (SL) differential equation:

$$
-\frac{d^{2}}{d x^{2}} y(x)+q(x) y(x)=\lambda y(x)
$$

where $\lambda \in \mathbb{C}$ is called the spectral parameter and the coefficient $q(x)$ is called the potential. In order to solve (1) using (2) one transforms [GGKM67 the potential $q(x)$ appearing in (2) to its "scattering data". Then one evolves with $t$ the scattering data using some simple differential equations. Finally, transforming back the evolved scattering data we obtain a new potential $q(x, t)$ of two variables, which solves (11) and satisfies $q(x, 0)=q(x)$. In other words, in this manner we solve the initial value problem for the equation (1). So, in order to solve (1) one has to find a "scattering data" for the given potential.

On the half line the question of characterizing of scattering data (or more precisely spectral measure $d \rho(\mu)$ on $\mathbb{R}$ ) for a given potential was completely solved for a continuously differentiable potential by Gelfand-Levitan theory GL51, but not always it can be used to solve the KdV equation (1). For this situation in case $\operatorname{var}[d \rho]<\infty$ a solution of $\mathrm{KdV}$ in the first quadrant $(x, t \geq 0)$ is presented in Mela].

Although there is a good scattering theory of the SL equation (2) on the line Mar77, Chapter 2, p. 128], [LS75, Chapter 2], even with arbitrary singularities [DZ91], the solutions of KdV, corresponding to them are not developed. In fact, the classes of initial potentials, for which solutions of (1) were presented using inverse scattering are as follows:

1. Soliton solutions correspond to $d \rho(\mu)$ to be a finite sum of point mass measures (discrete measure) Cru55,

2. Krichever solution Kri77, where $d \rho(\mu)=f(\mu) d \mu$ for $\mu \in \Gamma$ for some algebraic curve $\Gamma$,

3. Fadeyev inverse scattering theory $\mathrm{Fad74}$ where the $d \rho(\mu)$ is supported on the positive real line and has a finite number of point-mass measures on the negative real line,

4. Periodic potentials MW66] correspond to discrete spectral measures with accumulation point at infinity,

5. Quasi-periodic potentials [BJ02, DS75].

While analyzing the KdV equation (1) with an analytic initial potential, one can apply Taylour series techniques to try to solve the equation. It turns out that the corresponding combinatorial problem is extremely dificult. In fact a recent result of M. Goldstein and D. Damanik GD proving existence of a global solution of the KdV equation (1) with a quasi periodic potential involves an extremely sophisticated combinatorics of the powers of exponents, corresponding to all "harmonics". Still, the general problem of constructing a solution of the $\mathrm{KdV}$ equation for a given analytic potential has yet to be solved.

Using theory of vessels, we show that it is indeed a rare case that a solution of the KdV equation would exists on $\mathbb{R} \times\left[0, t_{0}\right)$ for some $t_{0}$. And the reason for this is that there is an operator of the 
form $\mathbb{X}(x, t)=I+T(x, t), I$ - identity, $T(x, t)$ - trace class, which is usually invertible for $t=0$ for all $x$, but fails to be invertible uniformly for all $x$ for whatsoever $t>0$ is. Theoretically it explains why there is no a theory on the existence of the local solution of the KdV equation for arbitrary analytic initial potential and only special cases ares solvable: for example, in the Faddeyev case the inverse of $\mathbb{X}(x, t)$ is uniformly bounded, so the continuous perturbation of the inverse will exist on $\left[0, t_{0}\right)$ (see Remarks). In fact, the existence of the solution $q(x, t)$ of (1) on $\mathbb{R} \times\left[0, t_{0}\right)$ implies that there exists a vessel on the same set (see Theorem 5.9). This actually means that the theory of vessels is a universal tool to study solutions of the $\mathrm{KdV}$ equation (1).

We present now the Main Theorem.

Main Theorem $\mathbf{5 . 4}$ Suppose that $q(x)$ is an analytic function on $\mathbb{R}$. There exists a KdV vessel, which exists on $\Omega \subseteq \mathbb{R}^{2}$. For each $x \in \mathbb{R}$ there exists $T_{x}>0$ such that $\{x\} \times\left[-T_{x}, T_{x}\right] \in \Omega$. The potential $q(x)$ is realized by the vessel for $t=0$.

The idea of the proof for this Theorem is simple and appears in Melf. Using simple algebra calculation, it is possible to show that constructing a collection $\mathfrak{V}_{K d V}$ of bounded operators and spaces $(\mathcal{H}$ - Hilbert space)

$$
\begin{aligned}
& \mathfrak{V}_{K d V}=\left[\begin{array}{cccc}
C(x) & A_{\zeta}, \mathbb{X}(x), A & B(x) & \sigma_{1}, \sigma_{2}, \gamma, \gamma_{*}(x) \\
\mathbb{C}^{2} & \mathcal{K} & \mathbb{C}^{2} & \Omega
\end{array}\right], \\
& B, C^{*}: \mathbb{C}^{2} \rightarrow \mathcal{H}, \quad A, \mathbb{X}, A_{\zeta}: \mathcal{H} \rightarrow \mathcal{H} \\
& \sigma_{1}, \sigma_{2}, \gamma, \gamma_{*}(x, t)-2 \times 2 \text { matrices, } \Omega \subseteq \mathbb{R}^{2} \\
& \frac{\partial}{\partial x} B \sigma_{1}=-\left(A B \sigma_{2}+B \gamma\right), \quad \frac{\partial}{\partial t} B=i A \frac{\partial}{\partial x} B, \\
& \frac{\partial}{\partial x} C^{*} \sigma_{1}=-\left(A_{\zeta}^{*} C^{*} \sigma_{2}+C^{*} \gamma\right), \quad \frac{\partial}{\partial t} C^{*}=i A_{\zeta}^{*} \frac{\partial}{\partial x} C^{*}, \\
& \frac{\partial}{\partial x} \mathbb{X}=B \sigma_{2} C, \quad \frac{\partial}{\partial t} \mathbb{X}=i A B \sigma_{2} C-i B \sigma_{2} C A_{\zeta}+i B \gamma C, \\
& A \mathbb{X}+\mathbb{X} A_{\zeta}+B \sigma_{1} C=0 \\
& \gamma_{*}=\gamma+\sigma_{2} C \mathbb{X}^{-1} B \sigma_{1}-\sigma_{1} C \mathbb{X}^{-1} B \sigma_{2} \text {. }
\end{aligned}
$$

which is called a regular $\mathbf{K d V}$ vessel on $\Omega$, where $\mathbb{X}(x, t)$ is invertible, we obtain a solution of (1) on $\Omega$ as follows. For

$$
\sigma_{1}=\left[\begin{array}{ll}
0 & 1 \\
1 & 0
\end{array}\right], \quad \sigma_{2}=\left[\begin{array}{ll}
1 & 0 \\
0 & 0
\end{array}\right], \quad \gamma=\left[\begin{array}{ll}
0 & 0 \\
0 & i
\end{array}\right]
$$

the function $\left(\left(x_{0}, t_{0}\right) \in \Omega\right)$

$$
q_{\mathfrak{V}}(x, t)=-2 \frac{\partial^{2}}{\partial x^{2}} \ln \operatorname{det}\left(\mathbb{X}^{-1}\left(x_{0}, t_{0}\right) \mathbb{X}(x, t)\right)
$$

is analytic in both variables on $\Omega$ and satisfies (11). The main contribution of this paper is that one can construct a vessel $\mathfrak{V}_{K d V}$ such that for $t=0$ it holds that $q_{\mathfrak{V}}(x, 0)=q(x)$ for arbitrary given analytic function $q(x)$. We use unbounded operators on Krein spaces in this case. Moreover, the operators are $A=i \mu$ and $A_{\zeta}=-i \mu-\int_{0}^{\infty} d \bar{\rho}(\mu)$. for a $2 \times 2$ measure $d \bar{\rho}$ on $[0, \infty)$, creating a Krein space $\mathcal{K}=L^{2}(d \bar{\rho})$ (see Section 2 for details), used instead of the Hilbert space $\mathcal{H}$ in the original definition. The main ingredients of this construction are node, prevessel and vessel. A node is the $(0,0)$ value of the vessel $\mathfrak{V}_{K d V}$, and prevessel is a globally defined object (for all $x, t \in \mathbb{R}$ ), which does not include the matrix $\gamma_{*}(x, t)$ (thus there is no need to demand existence of the inverse of $\mathbb{X}(x, t))$. These notions are fully studied at the text along with their properties. Finally, the tau function $\tau(x, t)=\operatorname{det}\left(\mathbb{X}^{-1}(0,0) \mathbb{X}(x, t)\right)$ defines the set $\Omega \subseteq \mathbb{R}^{2}$ where the vessel $\mathfrak{V}_{K d V}$ exists and, as a result, where the the solution of (1) exists as well. We show in Main Theorem 5.4 that $\{x\} \times\left[-T_{x}, T_{x}\right] \subseteq \Omega$ for some $T_{x}>0$, depending on $x$. 
From the Main Theorem 5.4 it follows that it is difficult to create a general construction of solutions of (1) on a strip $\mathbb{R} \times\left[0, t_{0}\right)$. Instead, the focus must be made on the problem of characterization of classes of functions $q(x)$, for which the matrix $\mathbb{X}(x, t)$ is invertible. The formula for the inverse, if it exists is also given in terms of the fundamental solutions of (1). It seems that the ideas, presented in this text also have a potential to be applied to the locally integrable case: $\int_{0}^{x} q(y) d y<\infty$ for all $x \in \mathbb{R}$.

Similar calculations can be carried over for the vessel parameters

$$
\sigma_{1}=I=\left[\begin{array}{ll}
1 & 0 \\
0 & 1
\end{array}\right], \quad \sigma_{1}=\frac{1}{2}\left[\begin{array}{cc}
1 & 0 \\
0 & -1
\end{array}\right], \quad \gamma=0=\left[\begin{array}{ll}
0 & 0 \\
0 & 0
\end{array}\right]
$$

which create solutions $y=\left[\begin{array}{ll}0 & 1\end{array}\right] \gamma_{*}(x, t)\left[\begin{array}{l}1 \\ 0\end{array}\right]$ of the evolutionary Non Linear Schrödinger (NLS) equation

$$
i y_{t}+y_{x x}+2|y|^{2} y=0 \text {. }
$$

The proof of this fact can be found in Meld. As a result, the ideas presented in this work can be used to prove a similar to Main Theorem 5.4 result for the evolutionary NLS equation.

Defining

$$
\sigma_{1}=\left[\begin{array}{lll}
0 & 0 & 1 \\
0 & 1 & 0 \\
1 & 0 & 0
\end{array}\right], \sigma_{2}=\left[\begin{array}{lll}
1 & 0 & 0 \\
0 & 0 & 0 \\
0 & 0 & 0
\end{array}\right], \gamma=\left[\begin{array}{ccc}
0 & 0 & 0 \\
0 & 0 & 1 \\
0 & -1 & 0
\end{array}\right]
$$

and

$$
\tilde{\sigma}_{1}=\sigma_{1}, \quad \widetilde{\sigma}_{2}=\left[\begin{array}{ccc}
0 & -i & 0 \\
i & 0 & 0 \\
0 & 0 & 0
\end{array}\right], \quad \widetilde{\gamma}=\left[\begin{array}{ccc}
0 & 0 & 0 \\
0 & 0 & 0 \\
0 & 0 & i
\end{array}\right],
$$

it was shown in Mele that the collection

$$
\mathfrak{V}_{\text {Bouss }}=\left(C(x, t), A_{\zeta}, \mathbb{X}(x, t), A, B(x, t) ; \sigma_{1}, \sigma_{2}, \gamma, \gamma_{*}(x, t), \tilde{\sigma}_{1}, \tilde{\sigma}_{2}, \widetilde{\gamma} ; \mathcal{H}, \mathbb{C}^{3} ; \Omega\right),
$$

where the operators $C(x, t): \mathcal{H} \rightarrow \mathbb{C}^{3}, A_{\zeta}, \mathbb{X}(x, t), A: \mathcal{H} \rightarrow \mathcal{H}, B(x, t): \mathbb{C}^{3} \rightarrow \mathcal{H}$ and a $3 \times 3$ matrix function $\gamma_{*}(x, t)$ satisfy

$$
\begin{aligned}
\frac{\partial}{\partial x} B & =-\left(A B \sigma_{2}+B \gamma\right) \sigma_{1}^{-1} & \frac{\partial}{\partial t} B & =-\left(A B \widetilde{\sigma}_{2}+B \widetilde{\gamma}\right) \widetilde{\sigma}_{1}^{-1}, \\
\frac{\partial}{\partial x} C & =\sigma_{1}^{-1}\left(\gamma C-\sigma_{2} C A_{\zeta}\right) & \frac{\partial}{\partial t} C & =\widetilde{\sigma}_{1}^{-1}\left(\widetilde{\gamma} C-\widetilde{\sigma}_{2} C A_{\zeta}\right), \\
\frac{\partial}{\partial x} \mathbb{X} & =B \sigma_{2} C & \frac{\partial}{\partial t} \mathbb{X} & =B \widetilde{\sigma}_{2} C, \\
0 & =A \mathbb{X}+\mathbb{X} A_{\zeta}+B \sigma_{1} C, & & \\
\gamma_{*} & =\gamma+\sigma_{2} C \mathbb{X}^{-1} B \sigma_{1}-\sigma_{1} C \mathbb{X}^{-1} B \sigma_{2}, & &
\end{aligned}
$$

is a Boussinesq vessels. When the operators are bounded the function

$$
q(x)=-\frac{3}{2} \frac{\partial^{2}}{\partial x^{2}} \ln (\tau(x, t))=-\frac{3}{2} \frac{\partial^{2}}{\partial x^{2}} \ln \operatorname{det}\left(\mathbb{X}^{-1}(0,0) \mathbb{X}(x, t)\right)
$$

satisfies the Boussinesq equation

$$
q_{t t}=\frac{\partial^{2}}{\partial x^{2}}\left[3 q_{x x}-12 q^{2}\right]
$$

on the set $\Omega \subseteq \mathbb{R}^{2}$, where $\mathbb{X}(x, t)$ is invertible (See Mele for details). A similar to Main Theorem 5.4 result can be proved for the Boussinesq equation (4) using ideas of this work. 


\section{Background on Krein space theory}

Let $(\mathcal{H},\langle\cdot, \cdot\rangle)$ be a Hilbert space. Let $\mathbb{X}$ be a self-adjoint bounded operator on $\mathcal{H}$. We define a sesquilinear form $[\cdot, \cdot]$ on $\mathcal{H}$ as $[u, v]=\langle\mathbb{X} u, v\rangle$. The map $[\cdot, \cdot]: \mathcal{H} \times \mathcal{H} \rightarrow \mathbb{C}^{2}$ possesses the following properties

1. $\left[\alpha x_{1}+\beta x_{2}, y\right]=\left[\alpha x_{1}, y\right]+\left[\beta x_{2}, y\right], x_{1}, x_{2}, y \in \mathcal{H}, \alpha, \beta \in \mathbb{C}$,

2. $[x, y]=\overline{[y, x]}, x, y \in \mathcal{H}$,

3. $|[x, y]| \leq C\|x\|\|y\|, x, y \in \mathcal{H}$ with $C>0$.

Here $\|\cdot\|$ denotes the Hilbert space norm of $\mathcal{H}$. Conversely, if there is a map $[\cdot, \cdot]$ with these three properties, there exists a self-adjoint operator $\mathbb{X}: \mathcal{H} \rightarrow \mathcal{H}$ realizing it by $[x, y]=\langle\mathbb{X} x, y\rangle$.

Let $\mathcal{K}$ be equal to the Hilbert space $\mathcal{H}$ as a set, equipped with (indefinite) inner product: $(\mathcal{K},[\cdot, \cdot])$. Then the pair $(\mathcal{K},[\cdot, \cdot])$ is called Krein space. For any operator $T$ on $\mathcal{K}$ we denote by $T^{*} 1$ the unique operator satisfying $[T u, v]=\left[u, T^{*} v\right]$ for all $u, v \in \mathcal{K}$. If we denote by $T^{<*>}$ the Hilbert space adjoint of $T$, and if $\mathbb{X}$ is invertible, then

$$
T^{*}=\mathbb{X}^{-1} T^{\langle *\rangle} \mathbb{X}
$$

The space $\mathcal{H}$ admits the decomposition

$$
\mathcal{H}=\mathcal{H}^{+} \oplus \mathcal{H}^{-}
$$

such that $[u, u]>0$ for all $x \in \mathcal{H}^{+}$and $[u, u]<0$ for all $x \in \mathcal{H}^{-}$. Moreover, the spaces $\left(\mathcal{H}^{+},[\cdot, \cdot]\right),\left(\mathcal{H}^{-},-[\cdot, \cdot]\right)$ are complete with respect to the norms $[\cdot, \cdot]$ and $-[\cdot, \cdot]$ respectively.

A typical example of a Krein space is as follows. If $\rho(\mu)$ is a real function which is locally of bounded variation and $|\rho(\mu)|$ denotes its total variation, then the space $L^{2}(\rho)$, of all measurable functions $f$ such that $\int_{\mathbb{R}}|f(\mu)|^{2} d|\rho(\mu)|<\infty$ and equipped with the indefinite inner product

$$
[f, g]=\int_{\mathbb{R}} g^{*}(\mu) f(\mu) d \rho(\mu),
$$

is a Krein space. A typical example used in this work involves a matrix-valued measure. Let $d \bar{\rho}=\left[\begin{array}{ll}d \rho_{11} & d \rho_{12} \\ d \rho_{21} & d \rho_{22}\end{array}\right]$ be a $2 \times 2$ matrix of measures. A complex-valued, self-adjoint $2,2 \times 2$ measure is called positive, if

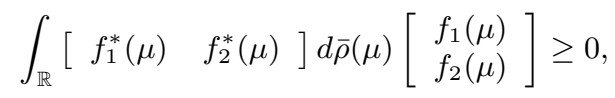

provided the integral exists. Denote by $\mathcal{R}=\operatorname{supp}(d \rho)$ - the support of the measure $d \rho$, then it is a matter of standard verifications that

$$
\mathcal{H}=L^{2}(d \bar{\rho})=\left\{\bar{f}=\left[\begin{array}{l}
f_{1}(\mu) \\
f_{2}(\mu)
\end{array}\right] \mid\|f\|^{2}=\int_{\mathcal{R}}\left[\begin{array}{ll}
f_{1}^{*}(\mu) & f_{2}^{*}(\mu)
\end{array}\right] d \bar{\rho}(\mu)\left[\begin{array}{l}
f_{1}(\mu) \\
f_{2}(\mu)
\end{array}\right]<\infty\right\}
$$

is a Hilbert space. Suppose that $d \bar{\rho}=d \bar{\rho}_{+}-d \bar{\rho}_{-}$for two positive measures $d \bar{\rho}_{+}, d \bar{\rho}_{-}$, creating two Hilbert spaces of column-functions $\mathcal{H}_{+}, \mathcal{H}_{-}$as above. We define

$$
\mathcal{K}=\left\{\bar{f}(\mu) \mid\|\bar{f}\|_{\mathcal{H}_{+}}+\|\bar{f}\|_{\mathcal{H}_{-}}<\infty\right\},
$$

\footnotetext{
${ }^{1}$ In the literature one usually denotes the adjoint of an operators $T$, with respect to $\langle\cdot, \cdot\rangle$ as $T^{*}$, and the adjoint with respect to $[\cdot, \cdot]$ as $T^{+}$. Since we are dealing exclusively with the Krein-space adjoint, we will use $T^{*}$ for this notation and will rarely denote by $T^{\langle *\rangle}$ the adjoint with respect to the Hilbert space $\mathcal{H}$.

${ }^{2}$ self-adjoint means $\rho_{11}=\rho_{11}^{*}, \rho_{12}=\rho_{21}^{*}, \rho_{22}=\rho_{22}^{*}$
} 
equipped with the indefinite inner product $\left(\mathcal{R}=\operatorname{supp}\left(d \rho_{+}\right) \cup \operatorname{supp}\left(d \rho_{-}\right)\right)$

$$
\begin{aligned}
& {[\bar{f}, \bar{g}]=\int_{\mathcal{R}}\left[\begin{array}{ll}
g_{1}^{*}(\mu) & g_{2}^{*}(\mu)
\end{array}\right] d \bar{\rho}(\mu)\left[\begin{array}{l}
f_{1}(\mu) \\
f_{2}(\mu)
\end{array}\right]}
\end{aligned}
$$

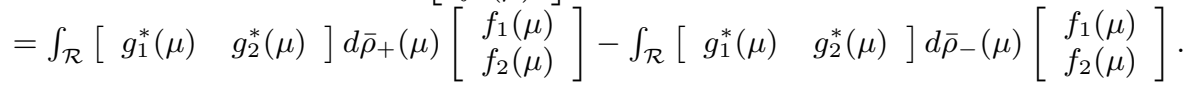

The space of all bounded operators between Krein space is denoted by $L\left(\mathcal{K}_{1}, \mathcal{K}_{2}\right)$. In this work we frequently use a 2 dimensional Hilbert space $\mathbb{C}^{2}$ for either $\mathcal{K}_{1}$ or $\mathcal{K}_{2}$. In this case we identify the sesquilinear form on $\mathbb{C}^{2}$ with the standard inner product of $\mathbb{C}^{2}$.

If $T: \mathcal{K}_{1} \rightarrow \mathcal{K}_{2}$ then its adjoint $T^{*}: \mathcal{K}_{2} \rightarrow \mathcal{K}_{1}$ is defined as the unique operator, satisfying

$$
[T x, y]_{\mathcal{K}_{2}}=\left[x, T^{*} y\right]_{\mathcal{K}_{1}} .
$$

We present class of operators, which generate analytic semi groups. An operator $A: \mathcal{K} \rightarrow \mathcal{K}$ (usually instead of $\mathcal{K}$ Banach spaces are used) generates an analytic semigroup if there exists $w>0$ such that $\Re \lambda>w$ is contained in the resolvent set of $A$ and there is $C>0$ such that

$$
\left\|(\lambda I-A)^{-1}\right\| \leq \frac{C}{|\lambda-w|} .
$$

The resolvent set of $A$ contains also the sector of the form

$$
\left.\operatorname{Sec}=\{\lambda \in \mathbb{C}|| \arg (\lambda)-w)<\frac{\pi}{2}+\delta\right\}
$$

for some $\delta>0$. Such generators possess "functional calculus":

$$
f(A)=\frac{1}{2 \pi i} \int_{\Gamma} f(\lambda)(\lambda I-A)^{-1} d \lambda,
$$

where $f(\lambda)$ is analytic in $S e c$ and the curve $\Gamma$ goes from $e^{-i \Theta_{0}} \infty$ to $e^{i \Theta_{0}} \infty$ entirely inside of $S e c$ (with $\frac{\pi}{2}<\Theta_{0}<\frac{\pi}{2}+\delta$ ). For example, the analytic semigroup, generated by the operator $A$ is

$$
e^{A x}=\frac{1}{2 \pi i} \int_{\Gamma} e^{\lambda x}(\lambda I-A)^{-1} d \lambda .
$$

We mention Hille-Yosida Theorem, characterizing generators of $C_{0}$ semigroups on $\mathbb{R}$, which is sufficient for some of the theorems.

Theorem 2.1. Let $A$ be a linear operator defined on a linear subspace $D(A)$ of the Banach space $\mathcal{K}, w$ be a real number, and $M>0$. Then A generates a strongly continuous semigroup, denoted as $e^{A x}$, that satisfies $\left\|e^{A x}\right\| \leq M e^{w x}$ if and only if

1. $D(A)$ is dense in $\mathcal{K}$, and

2. every real $\lambda>w$ belongs to the resolvent set of $A$ and for such $\lambda$ and for all positive integers $n$ :

$$
\left\|(\lambda I-A)^{-n}\right\| \leq \frac{M}{(\lambda-w)^{n}} .
$$

A proof of this Theorem can be found in Sta05, Theorem 3.4.1], EN00, Theorem II.3.5]. 


\section{$3 \quad$ Non-symmetric vessels}

Theory of operator nodes is presented in Bro71. We use a generalization of this notion, involving unbounded operators. This notion is used to study bounded operators $A=A_{R}+A_{I}=\frac{A+A^{*}}{2}+$ $\frac{A-A^{*}}{2 i}$, whose image part $A_{I}$ (or real part $A_{R}$ ) is small, or more precisely is compact. We substitute this requirement by the existence of $A_{\zeta}: \mathcal{H} \rightarrow \mathcal{H}$, such that $A+A_{\zeta}$ is 2-dimensional in a Krein space and these two operators have the same domain.

Many notions from the theory of nodes [Bro71] can be applied to the notion of a node, presented in this work. We have not inserted these results primarily for the lack of space, but also because of a different aim: we want to prove the existence of solutions for the KdV equation (11).

A prevessel is a node, for which some of the operators depend on $x \in \mathbb{R}$, and a vessel is an "invertible" prevessel (in the sense of Brodskii). The use of unbounded operators requires a careful consideration of their domains. The axioms of a node, presented here, assume equations, which take this issue into account.

\subsection{Node, prevessel, vessel}

Definition 3.1. A node is a collection of operators and spaces

$$
\mathfrak{N}=\left[\begin{array}{cccc}
C & A_{\zeta}, \mathbb{X}, A & B & \sigma_{1} \\
\mathbb{C}^{2} & \mathcal{K} & \mathbb{C}^{2} &
\end{array}\right]
$$

where $\mathcal{K}$ is a Krein space, $C: \mathcal{K} \rightarrow \mathbb{C}^{2}, \mathbb{X}: \mathcal{K} \rightarrow \mathcal{K}, B: \mathbb{C}^{2} \rightarrow \mathcal{K}$ are bounded operators, $\sigma_{1}=\sigma_{1}^{*}$ - invertible $2 \times 2$ matrix, $A, A_{\zeta}$ are generators of $C_{0}$ groups on $\mathcal{K}$ with identical dense domain $D(A)=D\left(A_{\zeta}\right)$. The operator $\mathbb{X}$ is assumed to satisfy $\mathbb{X}(D(A)) \subseteq D(A)$. The operators of the node are subject to the Lyapunov equation

$$
A \mathbb{X} u+\mathbb{X} A_{\zeta} u+B \sigma_{1} C u=0, \quad \forall u \in D\left(A_{\zeta}\right)=D(A) .
$$

If $\mathbb{X}$ is invertible, the transfer function of $\mathfrak{N}$ is

$$
S(\lambda)=I-C \mathbb{X}^{-1}(\lambda I-A)^{-1} B \sigma_{1} .
$$

The node $\mathfrak{N}$ is called symmetric if $A_{\zeta}=A^{*}$ and $C=B^{*}$.

Remarks: 1. a function $S(\lambda)$, representable in the form (7) is called realized [BGR90. 2. if $A_{\zeta}=A+T$ for a bounded operator $T$, then $D(A)=D\left(A_{\zeta}\right)\left(A_{\zeta}\right.$ is called a perturbation of $A$ in this case). 3. if $\mathbb{X}=I$, then the condition $\mathbb{X}(D(A)) \subseteq D(A)$ holds. 4. when the node is symmetric one can verify that

$$
S(\lambda) \sigma_{1}^{-1} S^{*}(-\bar{\lambda})=\sigma_{1}^{-1}
$$

at all points of analyticity of $S$. 5. for the unbounded operators $A, A_{\zeta}$ to be generators of $C_{0}$-groups, it necessary and sufficient to demand that they satisfy the conditions of the Hille-Yosida Theorem 2.1 Particularly, they must be closed, densely defined operators.

In the case $\mathbb{X}$ is invertible, we consider a stronger notion of a node as follows.

Definition 3.2. A node $\mathfrak{N}$ is called invertible, if $\mathbb{X}$ is invertible and $\mathbb{X}^{-1}(D(A)) \subseteq D(A)$.

A simple chain of inclusions for an invertible node

$$
\mathbb{X}(D(A)) \subseteq D(A) \Rightarrow D(A) \subseteq \mathbb{X}^{-1}(D(A)) \subseteq D(A),
$$


where the first inclusion comes from the node condition, and the last one from the invertible node condition, implies that $\mathbb{X}^{-1}(D(A))=D(A)$. Similarly, $\mathbb{X}(D(A))=D(A)$. Moreover, taking $u=$ $\mathbb{X}^{-1} u^{\prime}$, where $u, u^{\prime} \in D(A)$ and plugging it into the Lyapunov equation (6), we obtain that

$$
A_{\zeta} \mathbb{X}^{-1} u^{\prime}+\mathbb{X}^{-1} A u^{\prime}+\mathbb{X}^{-1} B \sigma_{1} C \mathbb{X}^{-1} u^{\prime}=0, \quad \forall u^{\prime} \in D(A),
$$

after multiplying by $\mathbb{X}^{-1}$ from the left. From the existence of this Lyapunov equation we obtain the following Lemma.

Lemma 3.1. If $\mathfrak{N}$ is an invertible node, then

$$
\mathfrak{N}_{-1}=\left[\begin{array}{cccc}
C \mathbb{X}^{-1} & A, \mathbb{X}^{-1}, A_{\zeta} & \mathbb{X}^{-1} B & \sigma_{1} \\
\mathbb{C}^{2} & \mathcal{K} & \mathbb{C}^{2}
\end{array}\right]
$$

is also a node.

One could consider a similar notion of "adjointable" node, for which the adjoint of the Lyapunov equation (6) would define a node, but we do not insert details here. Actually, there is a theory of construction of new such nodes from old ones, similarly to the theory presented in Bro71, BGR90. In the case $\mathbb{X}=I$ (the identity operator) we have a very well developed theory BL58] of (symmetric) nodes with $A_{\zeta}=A^{*}$, which has a finite dimensional real part: $A+A^{*}=-B \sigma_{1} B^{*}$.

Finally, rewriting the Lyapunov equation (8), of the invertible node as follows

$$
\left(-A_{\zeta}\right) \mathbb{X}^{-1} u^{\prime}+\mathbb{X}^{-1}(-A) u^{\prime}+\mathbb{X}^{-1} B \sigma_{1}^{-1} \sigma_{1}\left(-\sigma_{1} C\right) \mathbb{X}^{-1} u^{\prime}=0, \quad \forall u^{\prime} \in D(A)
$$

we arrive to the node

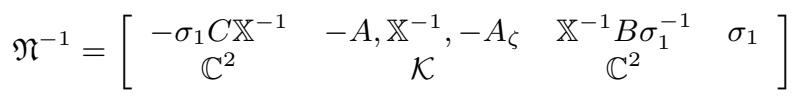

whose transfer unction

$$
S^{-1}(\lambda)=I+\sigma_{1} C\left(\lambda I+A_{\zeta}\right)^{-1} \mathbb{X}^{-1} B
$$

is the inverse of the transfer function, defined in (7), of the original invertible node $\mathfrak{N}$. This is a standard fact, related to Schur complements and can be found in Bro71, BGR90.

Definition 3.3. Class $\mathcal{R}\left(\sigma_{1}\right)$ consist of $2 \times 2$ matrix-valued functions $S(\lambda)$ of the complex variable $\lambda$, which are transfer functions of invertible nodes. The subclass $\mathcal{U}\left(\sigma_{1}\right) \subseteq \mathcal{R}\left(\sigma_{1}\right)$ consists of the transfer functions of symmetric, invertible nodes. The Schur class $\mathcal{S} \mathcal{U}\left(\sigma_{1}\right) \subseteq \mathcal{U}$ demand also that the inner space $\mathcal{K}$ is Hilbert and $\mathbb{X}>0$. The sub-classes of rational functions in $\mathcal{S U}, \mathcal{U}, \mathcal{R}$ are denoted by $\boldsymbol{r} \mathcal{S U}, \boldsymbol{r U}, \boldsymbol{r} \mathcal{R}$ respectively.

When $S(\lambda)$ is just analytic at infinity (hence $A$ must be bounded), there is a very well known theory of realizations developed in BGR90. For analytic at infinity and symmetric, i.e. satisfying $S^{*}(-\bar{\lambda}) \sigma_{1} S(\lambda)=\sigma_{1}$, functions there exists a good realization theory using Krein spaces, developed in $\mathrm{DLdS}^{3}$. Such a realization is then translated into a function in $\mathcal{U}\left(\sigma_{1}\right)$. The sub-classes $\mathcal{U}, \mathcal{S} \mathcal{U}$ appear a lot in the literature and correspond to the symmetric case. We will not particularly consider these two classes here and refer to AMV12.

Equations, which arise in the theory of vessels involve differential equations with unbounded operators. As a result, an operator satisfying such an equation must satisfy a relation with the domain of the unbounded operator, which is presented in the next Definition.

Definition 3.4. A bounded operator $B: \mathbb{C}^{2} \rightarrow \mathcal{K}$ is called $A$-regular, where $A: \mathcal{K} \rightarrow \mathcal{K}$ is linear, if $B e \in D(A)$ for all $e \in \mathbb{C}^{2}$.

\footnotetext{
${ }^{3}$ At the paper [DLdS a similar result is proved for functions symmetric with respect to the unit circle, but it can be translated using Calley transform into $S^{*}(-\bar{\lambda}) \sigma_{1} S(\lambda)=\sigma_{1}$ and was done in Melb AMV12
} 
Definition 3.5. Fix $2 \times 2$ matrices $\sigma_{2}=\sigma_{2}^{*}, \gamma=-\gamma^{*}$. The collection of operators and spaces

$$
\mathfrak{p r e} \mathfrak{V}=\left[\begin{array}{cccc}
C(x) & A_{\zeta}, \mathbb{X}(x), A & B(x) & \sigma_{1}, \sigma_{2}, \gamma \\
\mathbb{C}^{2} & \mathcal{K} & \mathbb{C}^{2} &
\end{array}\right]
$$

is called a (non-symmetric) prevessel, if the following conditions hold: 1 . prev is a node for all $x \in \mathbb{R}$, 2. the operator $B(x) \sigma_{2}$ is A-regular, 3. $C(x), \mathbb{X}(x), B(x)$ are differentiable, bounded operators, subject to the following conditions

$$
\begin{array}{r}
\frac{\partial}{\partial x} B(x)=-\left(A B \sigma_{2}+B \gamma\right) \sigma_{1}^{-1} \\
\frac{\partial}{\partial x} C(x) u=\sigma_{1}^{-1}\left(-\sigma_{2} C A_{\zeta} u+\gamma C u\right), \quad \forall u \in D\left(A_{\zeta}\right), \\
\frac{\partial}{\partial x} \mathbb{X}=B \sigma_{2} C,
\end{array}
$$

The prevessel prev is called symmetric if $A_{\zeta}=A^{*}$ and $C(x)=B^{*}(x)$ for all $x \in \mathbb{R}$.

It turns out that the structure of a prevessel implies the Lyapunov equations (6), (8) as the following Lemma claims.

Lemma 3.2 (permanence of the Lyapunov equations). Suppose that $B(x), C(x), \mathbb{X}(x)$ satisfy (10), (11), (12) respectively and $\mathbb{X}(x)\left(D\left(A_{\zeta}\right)\right) \subseteq D(A)$ for all $x \in \mathbb{R}$. Then if the Lyapunov equation (6) holds for a fixed $x_{0}$, then it holds for all $x$. In the case the operator $\mathbb{X}(x)$ is invertible and $B(x), C(x), \mathbb{X}(x)$ are part of an invertible node, if (8) holds for a fixed $x_{0}$, then it holds for all $x$.

Proof: Let us differentiate the right hand side of the Lyapunov equation (6):

$$
\begin{aligned}
& \frac{d}{d x}\left[A \mathbb{X} u+\mathbb{X} A_{\zeta} u+B \sigma_{1} C u\right]= \\
& =A B(x) \sigma_{2} C(x) u+B(x) \sigma_{2} C(x) A_{\zeta} u-A B(x) \sigma_{2} C(x) u-B(x) \sigma_{2} C(x) A_{\zeta} u \\
& =0
\end{aligned}
$$

The terms involving $\gamma$ are canceled, because $\gamma+\gamma^{*}=0$, by the assumption on it. Thus it is a constant and the result follows. For the invertible node case, the condition (8) is a result of (6).

Definition 3.6. The collection of operators, spaces and a set $\Omega \subseteq \mathbb{R}$

$$
\mathfrak{V}=\left[\begin{array}{cccc}
C(x) & A_{\zeta}, \mathbb{X}(x), A & B(x) & \sigma_{1}, \sigma_{2}, \gamma, \gamma_{*}(x) \\
\mathbb{C}^{2} & \mathcal{K} & \mathbb{C}^{2} & \Omega
\end{array}\right]
$$

is called a (non-symmetric) vessel, if $\mathfrak{V}$ is a pre-vessel, $\mathbb{X}(x)$ is invertible on $\Omega$, and $\mathfrak{V}$ is also an invertible node for all $x \in \Omega$. The $2 \times 2$ matrix-function $\gamma_{*}(x)$ satisfies the linkage condition on $\Omega$

$$
\gamma_{*}=\gamma+\sigma_{2} C \mathbb{X}^{-1} B \sigma_{1}-\sigma_{1} C \mathbb{X}^{-1} B \sigma_{2}
$$

The class of the transfer functions of vessels is defined as follows

Definition 3.7. Class $\mathcal{I}=\mathcal{I}\left(\sigma_{1}, \sigma_{2}, \gamma ; \Omega\right)$ consist of $2 \times 2$ matrix-valued (transfer) functions $S(\lambda, x)$ of the complex variable $\lambda$ and $x \in \Omega \subseteq \mathbb{R}$, possessing the following representation:

$$
S(\lambda, x)=I-C(x) \mathbb{X}^{-1}(x)(\lambda I-A)^{-1} B(x) \sigma_{1},
$$

where the operators $C(x), \mathbb{X}(x), B(x)$ are part of a vessel $\mathfrak{V}$.

Before we prove the Bäcklund transformation Theorem 3.4 we present a technical lemma. 
Lemma 3.3. Let $\mathfrak{V}$ be a vessel. Then for all $u \in D(A)$

$$
\begin{gathered}
\sigma_{1} \frac{d}{d x}\left[C(x) \mathbb{X}^{-1}(x)\right] u=\sigma_{2} C(x) \mathbb{X}^{-1}(x) A u+\gamma_{*}(x) C(x) \mathbb{X}^{-1}(x) u, \\
\frac{d}{d x}\left[\mathbb{X}^{-1}(x) B(x)\right] \sigma_{1}=A_{\zeta} \mathbb{X}^{-1}(x) B(x) \sigma_{2}-\mathbb{X}^{-1}(x) B(x) \gamma_{*}(x) .
\end{gathered}
$$

Proof: Consider (16) first. We write under each equality the corresponding equation that is used to derive the next line:

$$
\begin{aligned}
\sigma_{1} \frac{\partial}{\partial x}\left[C \mathbb{X}^{-1}\right] u= & \sigma_{1} \frac{\partial}{\partial x}(C) \mathbb{X}^{-1} u+\sigma_{1} C \frac{\partial}{\partial x}\left(\mathbb{X}^{-1}\right) u \\
& (\underline{112}): \quad \sigma_{1} \frac{\partial}{\partial x} C(x) u=-\sigma_{2} C A_{\zeta} u+\gamma C u \\
= & -\sigma_{2} C A_{\zeta} \mathbb{X}^{-1} u+\gamma C \mathbb{X}^{-1} u-\sigma_{1} C \mathbb{X}^{-1} B \sigma_{2} C \mathbb{X}^{-1} u \\
= & -\sigma_{2} C A_{\zeta} \mathbb{X}^{-1} u+\left(\gamma-\sigma_{1} C \mathbb{X}^{-1} B \sigma_{2}\right) C \mathbb{X}^{-1} u \\
& =\sigma_{2} C \mathbb{X}^{-1} A u+\left(\gamma+A_{\zeta} \mathbb{X}^{-1} u+\mathbb{X}^{-1} A u+\mathbb{X}^{-1} B \sigma_{1} C \mathbb{X}^{-1} u=0\right. \\
& \left(\underline{(14)}: \quad \gamma_{*}^{-1} B \sigma_{1}-\sigma_{1} C \mathbb{X}^{-1} B \sigma_{2}\right) C \mathbb{X}^{-1} u \\
= & \sigma_{2} C \mathbb{X}^{-1} A u+\gamma_{*} C \mathbb{X}^{-1} u .
\end{aligned}
$$

Notice that all equations of the vessel can be used, since we apply them to a vector $u$ from $D(A)$.

The equation (17) is proved in exactly the same manner.

Now we have all the ingredients of the following Theorem. This theorem has its origins at the work of M. Livşic Ls01 and was proved for bounded operators in Melb, Meld, AMV12. Now we present a generalization of these results for the case of unbounded operator $A$.

Theorem 3.4 (Vessel=Bäcklund transformation). Let $\mathfrak{V}$ be a vessel defined in (13) and satisfying the conditions of Definition [3.6. Fix $\lambda \notin \operatorname{spec}(A)$ and let $u(\lambda, x)$ be a solution of the input LDE

$$
\lambda \sigma_{2} u(\lambda, x)-\sigma_{1} \frac{\partial}{\partial x} u(\lambda, x)+\gamma u(\lambda, x)=0 .
$$

Then the function $y(\lambda, x)=S(\lambda, x) u(\lambda, x)$ satisfies the output $L D E$

$$
\lambda \sigma_{2} y(\lambda, x)-\sigma_{1} \frac{\partial}{\partial x} y(\lambda, x)+\gamma_{*}(x) y(\lambda, x)=0 .
$$

Proof: Let us fix $\lambda \notin \operatorname{spec}(A)$ and a solution $u(\lambda, x)$ of (18). Then for $y(\lambda, x)=S(\lambda, x) u(\lambda, x)$ we calculate:

$$
\begin{aligned}
\sigma_{1} \frac{d}{d x} y(\lambda, x)= & \sigma_{1} \frac{d}{d x}\left[\left(I-C(x) \mathbb{X}^{-1}(x)(\lambda I-A)^{-1} B(x) \sigma_{1}\right) u(\lambda, x)\right]= \\
= & \sigma_{1} \frac{d}{d x} u(\lambda, x)-\sigma_{1} \frac{d}{d x}\left[C(x) \mathbb{X}^{-1}(x)(\lambda I-A)^{-1} B(x) \sigma_{1} u(\lambda, x)\right] \\
= & \left(\sigma_{2} \lambda+\gamma\right) u(\lambda, x)-\sigma_{1} \frac{d}{d x}\left[C(x) \mathbb{X}^{-1}(x)\right](\lambda I-A)^{-1} B(x) \sigma_{1} u(\lambda, x) \\
& -\sigma_{1} C(x) \mathbb{X}^{-1}(x)(\lambda I-A)^{-1} \frac{d}{d x}[B(x)] \sigma_{1} u(\lambda, x) \\
& -\sigma_{1} C(x) \mathbb{X}^{-1}(x)(\lambda I-A)^{-1} B(x) \sigma_{1} \frac{d}{d x} u(\lambda, x) .
\end{aligned}
$$

Using (16), (10) and (18) it becomes (notice that $(\lambda I-A)^{-1} B(x) \sigma_{1} u(\lambda, x) \in D(A)$ )

$$
\begin{aligned}
& \sigma_{1} \frac{d}{d x} y(\lambda, x)=\left(\sigma_{2} \lambda+\gamma\right) u(\lambda, x)- \\
& -\left[\sigma_{2} C(x) \mathbb{X}^{-1}(x) A+\gamma_{*}(x) C(x) \mathbb{X}^{-1}(x)\right](\lambda I-A)^{-1} B(x) \sigma_{1} u(\lambda, x)+ \\
& \left.+\sigma_{1} C(x) \mathbb{X}^{-1}(x)(\lambda I-A)^{-1}\left[A B(x) \sigma_{2}+B(x) \not\right]\right] u(\lambda, x) \\
& \quad-\sigma_{1} C(x) \mathbb{X}^{-1}(x)(\lambda I-A)^{-1} B(x)\left(\sigma_{2} \lambda+\not 1\right) u(\lambda, x)=
\end{aligned}
$$


Let us combine the last two terms and add $\pm \lambda I$ next to $A$ :

$$
\begin{aligned}
& =\left(\sigma_{2} \lambda+\gamma\right) u(\lambda, x)- \\
& -\left[\sigma_{2} C(x) \mathbb{X}^{-1}(x)(A \pm \lambda I)+\gamma_{*}(x) C(x) \mathbb{X}^{-1}(x)\right](\lambda I-A)^{-1} B(x) \sigma_{1} u(\lambda, x)+ \\
& \quad+\sigma_{1} C(x) \mathbb{X}^{-1}(x)(\lambda I-A)^{-1}(A-\lambda I) B(x) \sigma_{2} u(\lambda, x)= \\
& =\left(\sigma_{2} \lambda+\gamma\right) u(\lambda, x)+\sigma_{2} C(x) \mathbb{X}^{-1}(x) B(x) \sigma_{1} u(\lambda, x)- \\
& -\left[\sigma_{2} C(x) \mathbb{X}^{-1}(x) \lambda+\gamma_{*}(x) C(x) \mathbb{X}^{-1}(x)\right](\lambda I-A)^{-1} B(x) \sigma_{1} u(\lambda, x)- \\
& \quad \quad-\sigma_{1} C(x) \mathbb{X}^{-1}(x) B(x) \sigma_{2} u(\lambda, x)= \\
& =\left(\sigma_{2} \lambda+\gamma+\sigma_{2} C(x) \mathbb{X}^{-1}(x) B(x) \sigma_{1}-\sigma_{1} C(x) \mathbb{X}^{-1}(x) B(x) \sigma_{2}\right) u(\lambda, x)- \\
& -\left[\sigma_{2} C(x) \mathbb{X}^{-1}(x) \lambda+\gamma_{*}(x) C(x) \mathbb{X}^{-1}(x)\right](\lambda I-A)^{-1} B(x) \sigma_{1} u(\lambda, x) .
\end{aligned}
$$

Using (14) and the definition of $S(\lambda, x)$ we obtain that

$$
\begin{aligned}
\sigma_{1} \frac{d}{d x} y(\lambda, x)= & {\left[\sigma_{2} \lambda+\gamma_{*}(x)\right] u(\lambda, x)-} \\
& {\left[\sigma_{2} \lambda-\gamma_{*}(x)\right] C(x) \mathbb{X}^{-1}(x)(\lambda I-A)^{-1} B(x) \sigma_{1} u(\lambda, x)=} \\
= & {\left[\sigma_{2} \lambda+\gamma_{*}(x)\right]\left[I-C(x) \mathbb{X}^{-1}(x)(\lambda I-A)^{-1} B(x) \sigma_{1}\right] u(\lambda, x)=} \\
= & \left(\sigma_{2} \lambda+\gamma_{*}(x)\right) S(\lambda, x) u(\lambda, x)= \\
= & \left(\sigma_{2} \lambda+\gamma_{*}(x)\right) y(\lambda, x) . \quad \square
\end{aligned}
$$

One of the corollaries CL55 of this Theorem is that the function $S(\lambda, x)$ must satisfy the following differential equation

$$
\frac{\partial}{\partial x} S(\lambda, x)=\sigma_{1}^{-1}\left(\sigma_{2} \lambda+\gamma_{*}(x)\right) S(\lambda, x)-S(\lambda, x) \sigma_{1}^{-1}\left(\sigma_{2} \lambda+\gamma\right) .
$$

Moreover, defining the fundamental solutions $\Phi(\lambda, x), \Phi_{*}(\lambda, x)$

$$
\begin{aligned}
\lambda \sigma_{2} \Phi(\lambda, x)-\sigma_{1} \frac{\partial}{\partial x} \Phi(\lambda, x)+\gamma \Phi(\lambda, x)=0, & \Phi(\lambda, 0)=I, \\
\lambda \sigma_{2} \Phi_{*}(\lambda, x)-\sigma_{1} \frac{\partial}{\partial x} \Phi_{*}(\lambda, x)+\gamma_{*}(x) \Phi_{*}(\lambda, x)=0, & \Phi_{*}(\lambda, 0)=I,
\end{aligned}
$$

we also obtain that

$$
S(\lambda, x)=\Phi_{*}(\lambda, x) S(\lambda, 0) \Phi^{-1}(\lambda, x)
$$

\subsection{Standard construction of a prevessel}

Now we present the standard construction of a prevessel prev from a node $\mathfrak{N}_{0}$. under assumption that the operators $A, A_{\zeta}$ are generators of analytic semi groups. In general, it is enough to demand that $A, A_{\zeta}$ possess "functional calculus". Using formula (5) and the fundamental matrices $\Phi(\lambda, x), \Phi_{*}(\lambda, x)$, defined in (21), (22) we make the following definition.

Definition 3.8. Let

$$
\mathfrak{N}_{0}=\left[\begin{array}{cccc}
C_{0} & A_{\zeta}, \mathbb{X}_{0}, A & B_{0} & \sigma_{1} \\
\mathbb{C}^{2} & \mathcal{K} & \mathbb{C}^{2} &
\end{array}\right]
$$

be a node, such that $A, A_{\zeta}$ generate analytic semi groups (or possess "functional calculus") and $D(A)=D\left(A_{\zeta}\right)$. The standard construction of the operators $B(x), C(x), \mathbb{X}(x)$ from the node $\mathfrak{N}_{0}$ 
is as follows

$$
\begin{array}{r}
B(x)=\frac{1}{2 \pi i} \int_{\Gamma}(\lambda I-A)^{-1} B_{0} \Phi^{*}\left(-\bar{\lambda}, x-x_{0}\right) d \lambda \\
C(x)=\frac{1}{2 \pi i} \int_{\Gamma} \Phi\left(\lambda, x-x_{0}\right) C_{0}\left(\lambda I+A_{\zeta}\right)^{-1} d \lambda \\
\mathbb{X}(x)=\mathbb{X}_{0}+\int_{x_{0}}^{x} B(y) \sigma_{2} C(y) d y .
\end{array}
$$

Theorem 3.5. The collection

$$
\mathfrak{p r e} \mathfrak{V}=\left[\begin{array}{cccc}
C(x) & A_{\zeta}, \mathbb{X}(x), A & B(x) & \sigma_{1}, \sigma_{2}, \gamma \\
\mathbb{C}^{2} & \mathcal{K} & \mathbb{C}^{2} &
\end{array}\right]
$$

defined by the standard construction from the node $\mathfrak{N}_{0}$ is a prevessel, coinciding with $\mathfrak{N}_{0}$ for $x=x_{0}$.

Proof: The condition $B(x) \sigma_{2}$ is $A$-regular comes from the definition of $B(x)$. Indeed, for all $\lambda, x$ $(\lambda I-A)^{-1} B_{0} \Phi\left(\lambda, x-x_{0}\right) \in D(A)$. By the existence of the functional calculus, it follows that (10), (11) hold. The equation (12) is immediate and the Lyapunov equation (6) follows from Lemma 3.2 Finally, we have to show that $\mathbb{X}(x)(D(A)) \subseteq D(A)$. For each $u \in D(A)$

$$
\mathbb{X}(x) u=\mathbb{X}_{0} u+\int_{x_{0}}^{x} B(y) \sigma_{2} C(y) u d y
$$

Here $\mathbb{X}_{0} u \in D(A)$ by the assumptions on $\mathfrak{N}_{0} . \quad B(y) \sigma_{2} \in D(A)$ by the $A$-regularity of $B(y) \sigma_{2}$. Moreover, since for each $u \in D(A)$

$$
\frac{\partial}{\partial x} B(x) \sigma_{1} C(x) u=-A B(x) \sigma_{2} C(x) u-B(x) \sigma_{2} C(x) A_{\zeta} u
$$

by integrating, we will obtain that

$$
\int_{x_{0}}^{x} A B(y) \sigma_{2} C(y) u d y=B_{0} \sigma_{1} C_{0} u-\frac{\partial}{\partial x} B(x) \sigma_{1} C(x) u+\int_{x_{0}}^{x} B(x) \sigma_{2} C(x) A_{\zeta} u d y
$$

exists. So, by the closeness of the operator $A$, we obtain that

$$
A \int_{x_{0}}^{x} B(y) \sigma_{2} C(y) u d y=\int_{x_{0}}^{x} A B(y) \sigma_{2} C(y) u d y
$$

exists and $\int_{x_{0}}^{x} B(y) \sigma_{2} C(y) u d y \in D(A)$.

If on an interval I, including $x_{0}$ the operator $\mathbb{X}(x)$ is also invertible, we can define $\gamma_{*}(x)$. In fact, the following Theorem holds.

Theorem 3.6 (local scattering). Suppose that $\mathfrak{N}_{0}$ is an invertible node, then there exists an interval $I$, including the given point $x_{0}$ and a vessel $\mathfrak{V}$ on $\mathrm{I}$, such that at $x=x_{0}$ the vessel $\mathfrak{V}$ coincides with the node $\mathfrak{N}_{0}$.

Proof: Since $\mathbb{X}_{0}$ is invertible, there exists a small interval I, including $x_{0}$ on which the operator

$$
\mathbb{X}(x)=\mathbb{X}_{0}+\int_{x_{0}}^{x} B(y) \sigma_{2} C(y) d y
$$


is invertible. As a result, we can define $\gamma_{*}(x)$ by the linkage condition (14). In order to show that the collection (13)

$$
\mathfrak{V}=\left[\begin{array}{cccc}
C(x) & A_{\zeta}, \mathbb{X}(x), A & B(x) & \sigma_{1}, \sigma_{2}, \gamma, \gamma_{*}(x) \\
\mathbb{C}^{2} & \mathcal{K} & \mathbb{C}^{2} & \mathrm{I}
\end{array}\right]
$$

is a vessel, it is necessary and sufficient to show that $\mathfrak{V}$ is an invertible node for all $x \in \mathrm{I}$, for which in turn we must show that $\mathbb{X}^{-1}(x)(D(A)) \subseteq D(A)$. Notice that

$$
\mathbb{X}^{-1}(x) u=\mathbb{X}_{0}^{-1} u-\int_{x_{0}}^{x} \mathbb{X}^{-1}(y) B(y) \sigma_{2} C(y) \mathbb{X}^{-1}(y) u d y
$$

and

$$
\begin{aligned}
& \frac{\partial}{\partial x}\left[\mathbb{X}^{-1}(x) B(x) \sigma_{1} C(x) \mathbb{X}^{-1}(x) u\right]= \\
& A_{\zeta} \mathbb{X}^{-1}(x) B(x) \sigma_{2} C(x) \mathbb{X}^{-1}(x) u+\mathbb{X}^{-1}(x) B(x) \sigma_{2} C(x) \mathbb{X}^{-1}(x) A,
\end{aligned}
$$

following from (16), (17). As a result, we can use the same proof as for $\mathbb{X}(x)(D(A)) \subseteq D(A)$ in Theorem 3.5

The transfer function

$$
S(\lambda)=I-C_{0} \mathbb{X}_{0}^{-1}(\lambda I-A)^{-1} B_{0} \sigma_{1}
$$

can be considered as a "scattering data", because $\gamma_{*}(x)$ (a generalized potential) is uniquely determined from $S(\lambda)$ by this construction. The uniqueness of $S(\lambda)$ for a given potential $\gamma_{*}(x)$ is false. For example, notice that multiplying the given intial value $S(\lambda)$ by arbitrary scalar function $a(\lambda)$, bounded at infinity, with limit 1 there, we will obtain that the two functions

$$
\Phi_{*}(\lambda, x) S(\lambda) \Phi^{-1}(\lambda, x), \quad a(\lambda) \Phi_{*}(\lambda, x) S(\lambda) \Phi^{-1}(\lambda, x)
$$

correspond to the same $\gamma_{*}(x)$. They can be obtained by applying the standard construction to $S(\lambda)$ and to $a(\lambda) S(\lambda)$.

A weaker form of the uniqueness is presented in the next Lemma. We emphasize that a similar Lemma was proved in the Sturm-Liouville case in [Melb] and analogous result exists in Fad74] for purely continuous spectrum.

Lemma 3.7. Suppose that two functions $S(\lambda, x), \widetilde{S}(\lambda, x)$ are in class $\mathcal{I}\left(\sigma_{1}, \sigma_{2}, \gamma ; \Omega\right)$, possessing the same initial value

$$
S(\lambda, 0)=\widetilde{S}(\lambda, 0)
$$

and are bounded at a neighborhood of infinity, with a limit value $I$ there. Then the corresponding outer potentials are equal on $\Omega$ :

$$
\gamma_{*}(x)=\widetilde{\gamma}_{*}(x)
$$

Proof: Suppose that

$$
S(\lambda, x)=\Phi_{*}(\lambda, x) S(\lambda, 0) \Phi^{-1}(\lambda, x), \quad \widetilde{S}(\lambda, x)=\widetilde{\Phi}_{*}(\lambda, x) S(\lambda, 0) \Phi^{-1}(\lambda, x),
$$

as in (23). Then

$$
\widetilde{S}^{-1}(\lambda, x) S(\lambda, x)=\widetilde{\Phi}_{*}(\lambda, x) \Phi_{*}^{-1}(\lambda, x)
$$

is entire (the singularities appear in $S(\lambda, 0)=\widetilde{S}(\lambda, 0)$ only and are canceled) and equal to $I$ (the identity matrix) at infinity. By a Liouville theorem, it is a constant function, namely $I$. So $\widetilde{\Phi}_{*}(\lambda, x) \Phi_{*}^{-1}(\lambda, x)=I$ or

$$
\widetilde{\Phi}_{*}(\lambda, x)=\Phi_{*}(\lambda, x) .
$$

If we differentiate this, we obtain that $\widetilde{\gamma}_{*}(x)=\gamma_{*}(x)$ on $\Omega$. 


\subsection{Tau function of a prevessel}

Existence of the vessel and its transfer function relies on the invertability of the operator $\mathbb{X}(x)$. In order to investigate the existence of the inverse for $\mathbb{X}(x)$ notice that from (12)

$$
\mathbb{X}(x)=\mathbb{X}_{0}+\int_{0}^{x} B(y) \sigma_{2} C(y) d y
$$

it follows that

$$
\mathbb{X}_{0}^{-1} \mathbb{X}(x)=I+\mathbb{X}_{0}^{-1} \int_{x_{0}}^{x} B(y) \sigma_{2} C(y) d y .
$$

Since $\sigma_{2}$ has finite rank, this expression is of the form $I+T$, for a trace class operator $T$ and since $\mathbb{X}_{0}$ is an invertible operator, there exists a non trivial interval (of length at least $\frac{1}{\left\|\mathbb{X}_{0}^{-1}\right\|}$ ) on which $\mathbb{X}(x)$ and $\tau(x)$ are defined. Recall GK69 that a function $F(x)$ from (a, b) into the group $\mathrm{G}$ (the set of bounded invertible operators on $\mathrm{H}$ of the form $\mathrm{I}+\mathrm{T}$, for a trace-class operator $T$ ) is said to be differentiable if $F(x)-I$ is differentiable as a map into the trace-class operators. In our case,

$$
\frac{d}{d x}\left(\mathbb{X}_{0}^{-1} \mathbb{X}(x)\right)=\mathbb{X}_{0}^{-1} \frac{d}{d x} \mathbb{X}(x)=\mathbb{X}_{0}^{-1} B(x) \sigma_{2} C(x)
$$

exists in trace-class norm. This leads us to the following

Definition 3.9. For a given prevessel prev (9) the tau function $\tau(x)$ is defined as

$$
\tau=\operatorname{det}\left(\mathbb{X}_{0}^{-1} \mathbb{X}(x)\right)
$$

Israel Gohberg and Mark Krein GK69, formula 1.14 on p. 163] proved that if $\mathbb{X}_{0}^{-1} \mathbb{X}(x)$ is a differentiable function into $\mathrm{G}$, then $\tau(x)=\operatorname{sp}\left(\mathbb{X}_{0}^{-1} \mathbb{X}(x)\right)$ is a differentiable map into $\mathbb{C}^{*}$ with

$$
\begin{aligned}
\frac{\tau^{\prime}}{\tau}=\operatorname{sp}\left(\left(\mathbb{X}_{0}^{-1} \mathbb{X}(x)\right)^{-1} \frac{d}{d x}\left(\mathbb{X}_{0}^{-1} \mathbb{X}(x)\right)\right) & =\operatorname{sp}\left(\mathbb{X}(x)^{\prime} \mathbb{X}^{-1}(x)\right)= \\
& =\operatorname{sp}\left(B(x) \sigma_{2} C(x) \mathbb{X}^{-1}(x)\right)=\operatorname{tr}\left(\sigma_{2} C(x) \mathbb{X}^{-1}(x) B(x)\right)
\end{aligned}
$$

Differentiating this expression, we obtain that

$$
\left(\frac{\tau^{\prime}}{\tau}\right)^{\prime}=\frac{\tau^{\prime \prime}}{\tau}-\left(\frac{\tau^{\prime}}{\tau}\right)^{2}=\frac{d}{d x} \operatorname{tr}\left(\sigma_{2} C(x) \mathbb{X}^{-1}(x) B(x)\right) .
$$

Using vessel conditions, since $B(x), \mathbb{X}^{-1}(x)$ are differentiable bounded operators in the case $A \mathbb{X}^{-1}(x) B(x)$ exists, or in the case it is canceled (SL case) we obtain that

$$
\begin{aligned}
& \frac{d}{d x} \operatorname{tr}\left(\sigma_{2} C(x) \mathbb{X}^{-1}(x) B(x)\right)= \\
& =\operatorname{tr}\left(\sigma_{2} \sigma_{1}^{-1}\left(-\sigma_{2} C(x) A_{\zeta}-\gamma^{*} C(x)\right) \mathbb{X}^{-1}(x) B(x)\right)- \\
& \quad-\operatorname{tr}\left(\sigma_{2} C(x) \mathbb{X}^{-1}(x) B(x) \sigma_{2} C(x) \mathbb{X}^{-1}(x) B(x)\right)+\operatorname{tr}\left(\sigma_{2} C(x) \mathbb{X}^{-1}(x)\left(-A B(x) \sigma_{2}-B(x) \gamma\right) \sigma_{1}^{-1}\right)= \\
& =\operatorname{tr}\left(\sigma_{2} \sigma_{1}^{-1} \sigma_{2} C(x)\left[-A_{\zeta} \mathbb{X}^{-1}-\mathbb{X}^{-1} A\right] B(x)\right)-\operatorname{tr}\left(\left[\sigma_{2} \sigma_{1}^{-1} \gamma^{*}+\gamma \sigma_{1}^{-1} \sigma_{2}\right] C(x) \mathbb{X}^{-1}(x) B(x)\right)- \\
& \quad-\operatorname{tr}\left(\sigma_{2} C(x) \mathbb{X}^{-1}(x) B(x) \sigma_{2} C(x) \mathbb{X}^{-1}(x) B(x)\right)= \\
& =\operatorname{tr}\left(\sigma_{2} \sigma_{1}^{-1} \sigma_{2} C(x) \mathbb{X}^{-1} B(x) \sigma_{1} C(x) \mathbb{X}^{-1} B(x)\right)-\operatorname{tr}\left(\left[\sigma_{2} \sigma_{1}^{-1} \gamma^{*}+\gamma \sigma_{1}^{-1} \sigma_{2}\right] C(x) \mathbb{X}^{-1}(x) B(x)\right)- \\
& \quad-\operatorname{tr}\left(\sigma_{2} C(x) \mathbb{X}^{-1}(x) B(x) \sigma_{2} C(x) \mathbb{X}^{-1}(x) B(x)\right) .
\end{aligned}
$$

\footnotetext{
${ }^{4} \mathrm{sp}$ - stands for the trace in the infinite dimensional space.
} 
Theorem 3.8. Suppose that $\mathfrak{p r e V}$ (9) is a prevessel. Define an open set

$$
\Omega=\{x \mid \tau(x) \neq 0\} .
$$

Then the prevessel pre $\mathfrak{V}$ is a vessel on $\Omega$.

Proof: for each $x_{0}$ in which $\tau\left(x_{0}\right) \neq 0$, the operator $\mathbb{X}\left(x_{0}\right)$ is invertible. Then there exists a closed interval $\mathrm{I}_{x_{0}}$, including $x_{0}$ on which prev defines a vessel by Theorem 3.6 . Then $\Omega=\cup_{x_{0}} \mathrm{I}_{x_{0}}$ and it finishes the proof.

\subsection{Moments and their properties}

If the function $S(\lambda)$ is analytic at the neighborhood of infinity, one can consider its Taylor series

$$
S(\lambda)=I-C \mathbb{X}^{-1}(\lambda I-A)^{-1} B \sigma_{1}=I-\sum_{n=0}^{\infty} \frac{H_{n}}{\lambda^{n+1}} \sigma_{1} .
$$

But in the general case this expansion may not converge. Still the following Definition can be in force.

Definition 3.10. The $n$-th moment $H_{n}$ of a vessel $\mathfrak{V}$ is:

$$
H_{n}=C \mathbb{X}^{-1} A^{n} B,
$$

provided that the image of $B$ is in $D\left(A^{n}\right)$. The moment is defined to be infinity, otherwise.

Moments will play a crucial role in the future research and we will the following defining property for them.

Theorem 3.9. Suppose that the moments $H_{0}, \ldots, H_{n+1}$ are finite and differentiable, then

$$
\left(H_{n}\right)_{x}^{\prime}=\sigma_{1}^{-1} \sigma_{2} H_{n+1}-H_{n+1} \sigma_{2} \sigma_{1}^{-1}+\sigma_{1}^{-1} \gamma_{*} H_{n}-H_{n} \gamma \sigma_{1}^{-1} .
$$

Proof: In the regular case, when all the operators are bounded, 31) follows from the differential equation (20). In the general case, it follows from (10), (12) and (14).

Theorem 3.10 (Uniqueness of the moments). Suppose that two sequences of moments $H_{n}(x)$ and $\widetilde{H}_{n}(x)$ are finite, differentiable and satisfy (35) with analytic $\gamma_{*}(x)$ and $\widetilde{\gamma}_{*}(x)$ respectively. Then from

$$
H_{n}(0)=\widetilde{H}_{n}(0), \quad \forall n=0,1,2, \ldots
$$

it follows that $\gamma_{*}(x)=\widetilde{\gamma}_{*}(x)$. If the infinite system of equations (35) has a unique sequence of solutions $H_{n}(x)$ for a given $\gamma_{*}(x)$ and initial values $H_{n}(0)$ then $H_{n}(x)=\widetilde{H}_{n}(x)$.

Proof: Let us show by the induction that $H_{0}^{(n)}(0)=(\widetilde{H})_{0}^{(n)}(0)$ for all $n=0,1,2, \ldots$. And since these two moments are analytic, the result will follow from the uniqueness of the Taylor series. For $n=0, H_{0}(0)=\widetilde{H}_{0}(0)$ and the basis of the induction follows. Then from (31) it follows that

$$
H_{0}^{(1)}(x)=\sigma_{1}^{-1} \sigma_{2} H_{1}-H_{1} \sigma_{2} \sigma_{1}^{-1}+\sigma_{1}^{-1} \gamma_{*} H_{0}-H_{0} \gamma \sigma_{1}^{-1} .
$$

Differentiating again, using (31) for $n=0,1$ and the Linkage condition (14), we will obtain that

$$
\begin{aligned}
& H_{0}^{(1)}(x)=\sigma_{1}^{-1} \sigma_{2} H_{1}-H_{1} \sigma_{2} \sigma_{1}^{-1}+\sigma_{1}^{-1} \gamma_{*} H_{0}-H_{0} \gamma \sigma_{1}^{-1}= \\
= & \sigma_{1}^{-1} \sigma_{2} H_{1}-H_{1} \sigma_{2} \sigma_{1}^{-1}+\sigma_{1}^{-1}\left(\gamma+\sigma_{2} H_{0} \sigma_{1}-\sigma_{1} H_{0} \sigma_{2}\right) H_{0}-H_{0} \gamma \sigma_{1}^{-1}=P_{2}\left(H_{0}(x), H_{1}(x), H_{2}(x)\right)
\end{aligned}
$$


for a non-commutative polynomial $P_{2}$ with constant coefficients (depending on $\sigma_{1}, \sigma_{1}, \gamma$ ). This shows that a simple induction results in

$$
H_{0}^{(n)}(x)=P_{n}\left(H_{0}(x), H_{1}(x), \ldots, H_{n}(x), H_{n+1}(x)\right)
$$

for a non-commutative polynomial $P_{n}$ with constant matrix-coefficients. As a result, plugging here $x=0$ and using the condition $H_{n}(0)=\widetilde{H}_{n}(0)$

$$
\begin{aligned}
H_{0}^{(n)}(0)=P_{n}\left(H_{0}(0), H_{1}(0), \ldots, H_{n}(0),\right. & \left.H_{n+1}(0)\right)= \\
& =P_{n}\left(\widetilde{H}_{0}(0), \widetilde{H}_{1}(0), \ldots, \widetilde{H}_{n}(0), \widetilde{H}_{n+1}(0)\right)=(\widetilde{H})_{0}^{(n)}(0) .
\end{aligned}
$$

From here it follows that $H_{0}(x)=\widetilde{H}_{0}(x)$ and hence by the linkage condition (14) $\gamma_{*}(x)=\widetilde{\gamma}_{*}(x)$. Then the last statement of the Theorem follows from the uniqueness of solutions.

\section{Sturm-Liouville vessels}

In the special case of SL vessel parameters, we obtain that equations (18), (19) are equivalent to (2). Let us explain it in more details.

Definition 4.1. The Sturm Liouville (SL) vessel parameters are defined as follows

$$
\sigma_{1}=\left[\begin{array}{ll}
0 & 1 \\
1 & 0
\end{array}\right], \quad \sigma_{2}=\left[\begin{array}{ll}
1 & 0 \\
0 & 0
\end{array}\right], \quad \gamma=\left[\begin{array}{ll}
0 & 0 \\
0 & i
\end{array}\right] .
$$

Suppose that we are given a SL vessel $\mathfrak{V}$, in other words, $\mathfrak{V}$ (13) is defined for the SL vessel parameters. Denote a differentiable $2 \times 2$ matrix function $H_{0}=B^{*}(x) \mathbb{X}^{-1}(x) B(x)=\left[\begin{array}{cc}a & b \\ c & d\end{array}\right]$. Then from (28) it follows that $\frac{\tau^{\prime}}{\tau}=\operatorname{tr}\left(\sigma_{2} B^{*}(x) \mathbb{X}^{-1}(x) B(x)\right)=a$ is the 1,1 entry of $H_{0}$. Using simple calculations it follows that

$$
\sigma_{2} \sigma_{1}^{-1} \sigma_{2}=0, \quad \operatorname{tr}\left(\sigma_{2} H_{0} \sigma_{2} H_{0}\right)=\left(\operatorname{tr}\left(\sigma_{2} H_{0}\right)\right)^{2}, \quad \sigma_{2} \sigma_{1}^{-1} \gamma^{*}+\gamma \sigma_{1}^{-1} \sigma_{2}=\left[\begin{array}{cc}
0 & -i \\
i & 0
\end{array}\right],
$$

and we obtain from (29) that

$$
\frac{\tau^{\prime \prime}}{\tau}=-\operatorname{tr}\left(\left[\begin{array}{cc}
0 & -i \\
i & 0
\end{array}\right] H_{0}\right)=i(c-b)
$$

Notice that the terms involving operator $A$ are canceled. Moreover, we obtain that

$$
\gamma_{*}(x)=\gamma+\sigma_{2} H_{0} \sigma_{1}-\sigma_{1} H_{0} \sigma_{2}=\left[\begin{array}{cc}
b-c & a \\
-a & i
\end{array}\right]=\left[\begin{array}{cc}
i \frac{\tau^{\prime \prime}}{\tau} & \frac{\tau^{\prime}}{\tau} \\
-\frac{\tau^{\prime}}{\tau} & i
\end{array}\right] .
$$

Thus we obtain the following lemma (appearing already in Mel11, Proposition 3.2 ])

Lemma 4.1. For $S L$ vessel parameters, the following formula for $\gamma_{*}(x)$ holds

$$
\gamma_{*}(x)=\left[\begin{array}{cc}
i \frac{\tau^{\prime \prime}}{\tau} & \frac{\tau^{\prime}}{\tau} \\
-\frac{\tau^{\prime}}{\tau} & i
\end{array}\right]
$$


Analogously to [Mel11, Section 3.1.1], simple calculations show that denoting $u(\lambda, x)=\left[\begin{array}{l}u_{1}(\lambda, x) \\ u_{2}(\lambda, x)\end{array}\right]$ we shall obtain that the input compatibility condition (18) is equivalent to

$$
\left\{\begin{array}{l}
-\frac{\partial^{2}}{\partial x^{2}} u_{1}(\lambda, x)=-i \lambda u_{1}(\lambda, x), \\
u_{2}(\lambda, x)=-i \frac{\partial}{\partial x} u_{1}(\lambda, x) .
\end{array}\right.
$$

The output $y(\lambda, x)=\left[\begin{array}{l}y_{1}(\lambda, x) \\ y_{2}(\lambda, x)\end{array}\right]=S(\lambda, x) u(\lambda, x)$ satisfies the output equation (19), which is equivalent to

$$
\left\{\begin{array}{l}
-\frac{\partial^{2}}{\partial x^{2}} y_{1}(\lambda, x)-2 \frac{d^{2}}{d x^{2}}[\ln (\tau(x))] y_{1}(\lambda, x)=-i \lambda y_{1}(\lambda, x), \\
y_{2}(\lambda, x)=-i\left[\frac{\partial}{\partial x} y_{1}(\lambda, x)+\frac{\tau^{\prime}}{\tau} y_{1}(\lambda, x)\right] .
\end{array}\right.
$$

Observing the first coordinates $u_{1}(\lambda, x), y_{1}(\lambda, x)$ of the vector-functions $u(\lambda, x), y(\lambda, x)$ we can see that multiplication by $S(\lambda, x)$ maps solution of the trivial SL equation (i.e. $q(x)=0)$ to solutions of the more complicated one, defined by the potential

$$
q(x)=-2 \frac{d^{2}}{d x^{2}}[\ln (\tau(x))]
$$

which can be considered as an analogue of the scattering theory.

\subsection{Construction of a realized function, possessing given Moments}

Notice that the formula for the first moment is

$$
H_{0}=\left[\begin{array}{cc}
\frac{\tau^{\prime}}{\tau} & b \\
c & h_{0}^{22}
\end{array}\right], \quad i(b-c)=\frac{\tau^{\prime \prime}}{\tau} .
$$

In the case $c=-b$, we obtain the following form, which will be used further in the text:

$$
H_{0}=\left[\begin{array}{cc}
\frac{\tau^{\prime}}{\tau} & -\frac{i \tau^{\prime \prime}}{2 \tau} \\
\frac{i \tau^{\prime \prime}}{2 \tau} & h_{0}^{22}
\end{array}\right]
$$

Studying equation (31) one can obtain a formula for the moment $H_{n+1}=\left[\begin{array}{cc}H_{n+1}^{11} & H_{n+1}^{12} \\ H_{n+1}^{21} & H_{n+1}^{22}\end{array}\right]$ in terms of $H_{n}=\left[\begin{array}{ll}H_{n}^{11} & H_{n}^{12} \\ H_{n}^{21} & H_{n}^{22}\end{array}\right]$ as follows (here $\beta=-\frac{\tau^{\prime}}{\tau}, \pi_{11}=\beta^{\prime}-\beta^{2}$ ):

$$
\begin{cases}H_{n+1}^{11} & =i H_{n}^{22}-\frac{d}{d x} H_{n}^{12}+\beta H_{n}^{12} \\ H_{n}^{12}-H_{n}^{21} & =i\left(\frac{d}{d x} H_{n+1}^{11}-\beta H_{n+1}^{11}\right) \\ \frac{d}{d x}\left(H_{n}^{12}+H_{n}^{21}\right) & =-i \pi_{11} H_{n}^{11}+\beta\left(H_{n}^{12}-H_{n}^{21}\right) \\ 2 i \frac{d}{d x} H_{n}^{22} & =\frac{d^{2}}{d x^{2}} H_{n}^{12}-2 \beta \frac{d}{d x} H_{n}^{12} .\end{cases}
$$

Indeed, plugging the SL vessel parameters (Definition 4.1) into (31) we find that

$$
\begin{aligned}
\frac{d}{d x}\left[\begin{array}{cc}
H_{n}^{11} & H_{n}^{12} \\
H_{n}^{21} & H_{n}^{22}
\end{array}\right] & =\sigma_{1}^{-1} \sigma_{2} H_{n+1}-H_{n+1} \sigma_{2} \sigma_{1}^{-1}+\sigma_{1}^{-1} \gamma_{*} H_{n}-H_{n} \gamma \sigma_{1}^{-1} \\
& =\left[\begin{array}{cc}
0 & -H_{n+1}^{11} \\
H_{n+1}^{11} & H_{n+1}^{12}-H_{n+1}^{21}
\end{array}\right]+\left[\begin{array}{cc}
\beta H_{n}^{11}+i \beta\left(H_{n}^{21}-H_{n}^{12}\right) & \beta H_{n}^{12}+i H_{n}^{22} \\
-i \pi_{11} H_{n}^{11}-\beta H_{n}^{21}-H_{n}^{22} & -i \pi_{11} H_{n}^{12}-\beta H_{n}^{22}
\end{array}\right]
\end{aligned}
$$


Expressing $H_{n+1}^{12}$ from 1,2 and 2,1 entries of this equality, we obtain that

$$
H_{n+1}^{11}=-\frac{d}{d x} H_{n}^{12}+\beta H_{n}^{12}+i H_{n}^{22}=\frac{d}{d x} H_{n}^{21}-\left(-i \pi_{11} H_{n}^{11}-\beta H_{n}^{21}-i H_{n}^{22}\right)
$$

The first equality here is identical to the first equality in (35), and the second equality is identical to the third line of (35). In a similar manner one can derive the other equations (see [AMV] for more details).

From the formulas (35) it follows that we can construct the moments $H_{n}$ with a special pattern. Namely, one can choose the initial conditions for $H_{n}^{12}+H_{n}^{21}$ and $H_{n}^{22}$ (which are not assigned in (35) ) so that the following Lemma holds.

Lemma 4.2. There exists a choice of initial conditions such that

$$
H_{n}=i^{n}\left[\begin{array}{cc}
r_{n}^{11} & i b_{n}^{12} \\
-i b_{n}^{12} & d_{n}^{22}
\end{array}\right]
$$

with real-valued function $r_{n}^{11}, b_{n}^{12}, d_{n}^{22}$. More precisely, the conditions at 0 must be chosen so that

$$
i^{n} H_{n}^{22}(0) \in \mathbb{R}, \quad H_{n}^{12}+H_{n}^{21}=0 .
$$

Proof: Using induction, it is necessary to choose $r=0, h_{0}^{22} \in \mathbb{R}$ in formula (34) and the first moment $H_{0}$ will satisfy the required condition. Suppose that $H_{n}$ is of the form stated at the theorem. Then from (35) it follows that $H_{n+1}^{11}=i^{n+1}\left(d_{n}^{22}-\frac{d}{d x} r_{n}^{11}+\beta r_{n}^{11}\right)=i^{n+1} r_{n+1}^{11}$ and is of the required form. Similarly, the other formulas of (35) produce the required result. Notice that the initial conditions for $H_{n+1}$ must be chosen so that $i^{n+1} H_{n+1}^{22}(0) \in \mathbb{R}$ and $H_{n+1}^{12}(0)+H_{n+1}^{21}(0)=0$.

Suppose that we are given moments $H_{n}$, realized in the form (36). We would like to construct a function $S(\lambda)$ with these moments, analytic at $\mathbb{C} \backslash i \mathbb{R}$ and possessing the following realization form

$$
S(\lambda)=I-\int_{\mathbb{R}} \frac{1}{\lambda-i \mu} d \bar{\rho}(\mu) \sigma_{1},
$$

where $d \bar{\rho}=d \bar{\rho}_{+}-d \bar{\rho}_{-}$is a $2 \times 2$ matrix measure, which is a difference of two positive matrixmeasures $d \bar{\rho}_{+}, d \bar{\rho}_{-}$. Moreover, we want the measures $\bar{\rho}, d \bar{\rho}_{+}, d \bar{\rho}_{-}$to be analytic, namely, to satisfy the following

Definition 4.2. A measure $d \bar{\rho}$ is called analytic, if $\int_{\mathbb{R}} \mu^{n} d \bar{\rho}(\mu)$ is finite for each $n=0,1,2, \ldots$ For each function $S(\lambda)$, realized in the form (37) with an analytic measure $\bar{\rho}$, we define its $n$-th moment as $H_{n}^{S}=\int_{\mathbb{R}}(i \mu)^{n} d \bar{\rho}$.

Theorem 4.3. Given a sequence of moments $H_{n}$ of the form (36), there exists a function $S(\lambda)$, realized in form (37) with an analytic measure $d \bar{\rho}=\left[\begin{array}{cc}d \rho_{11} & i d \rho_{12} \\ -i d \rho_{12} & d \rho_{22}\end{array}\right]$ on $\mathbb{R}$, with real signed measures $\rho_{11}, \rho_{12}, \rho_{22}$ whose moments coincide with the given ones. Namely, it holds that $H_{n}^{S}=H_{n}$.

Remark: this Theorem is best illustrated if $d \bar{\rho}$ has a bounded support. Taking $\lambda$, satisfying $|\lambda|>\sup |\operatorname{supp}(d \rho)|$, there exists Taylor expansion $\frac{1}{\lambda-i \mu}=\sum_{n=0}^{\infty} \frac{(i \mu)^{n}}{\lambda^{n+1}}$ and the moments $H_{n}^{S}$ are just the Taylor coefficients. This also gives an idea of the converse construction, when given moments $H_{n}$ have exponential growth: $\left\|H_{n}\right\| \leq k C^{n}$. Define the function as $S(\lambda)=I-\sum_{n=0}^{\infty} \frac{H_{n}}{\lambda^{n+1}} \sigma_{1}$. The fact that this function can be extended to $\mathbb{C} \backslash i R$ with a realization in the form (37) follows from this theorem. 
Proof: We use the Hamburger problem, which constructs a positive Borel measure $\rho$ for a given set of real numbers $m_{0}, m_{1}, m_{2}, \ldots$. More precisely, there exists a Borel measure $\rho$ satisfying $\int_{\mathbb{R}} \mu^{n} d \rho(\mu)=m_{n}$ if and only if the Hankel matrices

$$
M_{n}=\left[\begin{array}{cccc}
m_{0} & m_{1} & \ldots & m_{n} \\
m_{1} & m_{2} & \ldots & m_{n+1} \\
\vdots & \vdots & \ddots & \vdots \\
m_{n} & m_{n+1} & \ldots & m_{2 n}
\end{array}\right]
$$

are positive.

In order to prove our theorem, notice that it is enough to prove the scalar case, because the $2 \times 2$ case consists of four problems for each entry:

$$
H_{n}^{S}=i^{n} \int_{\mathbb{R}} \mu^{n} d \bar{\rho}(\mu)=i^{n} \int_{\mathbb{R}} \mu^{n}\left[\begin{array}{cc}
d \rho_{11} & i d \rho_{12} \\
-i d \rho_{12} & d \rho_{22}
\end{array}\right]=i^{n}\left[\begin{array}{cc}
r_{n}^{11} & i b_{n}^{12} \\
-i b_{n}^{12} & d_{n}^{22}
\end{array}\right]=H_{n},
$$

or equivalently

$$
\int_{\mathbb{R}} \mu^{n} d \rho_{11}=r_{n}^{11}, \quad \int_{\mathbb{R}} \mu^{n} d \rho_{12}=b_{n}^{12}, \quad \int_{\mathbb{R}} \mu^{n} d \rho_{22}=d_{n}^{22} .
$$

The scalar problem is solvable as follows. Suppose that we are given a set of real numbers $m_{0}, m_{1}, m_{2}, \ldots$. Let $M_{n}$ be the corresponding Hankel matrices. We are going to construct two additional sequences $v_{n}, u_{n}$ which satisfy the conditions of the Hamburger Theorem and are such that $m_{n}=v_{n}-u_{n}$. For $m_{0}$, it is immediate that $m_{0}=v_{0}-u_{0}$, for some positive $v_{0} \neq 0$ and $u_{0}$. Suppose by induction, that we have constructed $v_{1}, \ldots, v_{2 n}, u_{1}, \ldots, u_{2 n}$ and matrices $V_{n}, U_{n}$, similar to the construction of $M_{n}$. Suppose also by the induction that $\operatorname{det} V_{n}>0$. Let $v_{2 n+1}, v_{2(n+1)}$ be parameters and construct next

$$
M_{n+1}=\left[\begin{array}{cc}
M_{n} & \bar{m} \\
\bar{m}^{t} & m_{2(n+1)}
\end{array}\right], \quad V_{n+1}=\left[\begin{array}{cc}
V_{n} & \bar{v} \\
\bar{v}^{t} & v_{2(n+1)}
\end{array}\right], \quad U_{n+1}=V_{n+1}-M_{n+1},
$$

where $\bar{m}=\left[\begin{array}{c}m_{n+1} \\ m_{n+2} \\ \vdots \\ m_{2 n+1}\end{array}\right], \bar{v}=\left[\begin{array}{c}v_{n+1} \\ v_{n+2} \\ \vdots \\ v_{2 n+1}\end{array}\right]$. Using the principal minors criteria for the positivity of a matrix, all the principal minors of $V_{n+1}$ are those of $V_{n}$, except for the last one:

$$
\operatorname{det}\left(V_{n+1}\right)=v_{2(n+1)} \operatorname{det} V_{n}+C_{1} .
$$

The last formula is obtained by expansion along the last column of $V_{n+1}$. Taking $v_{2(n+1)}>$ $-\frac{C_{1}}{\operatorname{det}\left(V_{n}\right)}$, we obtain that $V_{n+1}$ is positive and $\operatorname{det} V_{n+1}>0$. Similarly, all the principal minors of $U_{n+1}$ are those fo $U_{n}$ (hence positive), except for the last one:

$$
\operatorname{det} U_{n+1}=\left(v_{2(n+1)}-m_{2(n+1)}\right) \operatorname{det} U_{n}+C_{2},
$$

so we have to demand that $v_{2(n+1)}>m_{2(n+1)}+\frac{C_{2}}{\operatorname{det} U_{n}}$ resulting in the positivity of the matrix $U_{n+1}$ and $\operatorname{det} U_{n+1}>0$. Notice that $v_{2 n+1}$ is arbitrary and we choose $v_{2(n+1)}>\max \left(-\frac{C_{1}}{\operatorname{det}\left(V_{n}\right)}, m_{2(n+1)}+\right.$ $\left.\frac{C_{2}}{\operatorname{det} U_{n}}\right)$, finishing the construction of the sequences $v_{n}, u_{n}$. 
Finally, using Hamburger theorem, we find measures $\rho_{+}$and $\rho_{-}$such that

$$
v_{n}=\int_{\mathbb{R}} \mu^{n} d \rho_{+}(\mu), \quad u_{n}=\int_{\mathbb{R}} \mu^{n} d \rho_{-}(\mu) .
$$

As a result, for the signed measure $d \rho=d \rho_{+}-d \rho_{-}$it holds that

$$
\int_{\mathbb{R}} \mu^{n} d \rho=\int_{\mathbb{R}} \mu^{n} d \rho_{+}-\int_{\mathbb{R}} \mu^{n} d \rho_{-}=v_{n}-u_{n}=m_{n}
$$

Next theorem appears in Boa39.

Corollary 4.4. Given a sequence of moments $H_{n}$ of the form (36), there exists a function $S(\lambda)$, realized in form (37) with an analytic measure $d \bar{\rho}=\left[\begin{array}{cc}d \rho_{11} & i d \rho_{12} \\ -i d \rho_{12} & d \rho_{22}\end{array}\right]$ as in Theorem 4.3 with support on $[0, \infty]$.

Proof: Using the construction of Theorem 4.3 we can add a requirement on the choice of $v_{2 n+1}, u_{2 n+1}$ so that the conditions of the Stieltjes moment problem are fulfilled. Additionally to the positivity of $V_{n}$ one has to require also that the matrices

$$
V_{n}^{\prime}=\left[\begin{array}{cccc}
v_{1} & v_{2} & \ldots & v_{n+1} \\
v_{2} & v_{2} & \ldots & v_{n+2} \\
\vdots & \vdots & \ddots & \vdots \\
v_{n+1} & v_{n+2} & \ldots & v_{2 n+1}
\end{array}\right]
$$

are positive (similar matrix $U_{n}^{\prime}$ is constructed from $u_{n}$ 's). The condition is easily obtained when one considers the principal minors and uses the induction similarly to the proof of Theorem 36

Theorem 4.5. Suppose that a function $S(\lambda)$ possesses a realization (37)

$$
S(\lambda)=I-\int_{\mathbb{R}} \frac{1}{\lambda-i \mu} d \bar{\rho}(\mu) \sigma_{1}
$$

for an analytic measure $d \bar{\rho}=\left[\begin{array}{cc}d \rho_{11} & i d \rho_{12} \\ -i d \rho_{12} & d \rho_{22}\end{array}\right]$. Then there exists an invertible node

$$
\mathfrak{N}_{0}=\left[\begin{array}{cccc}
C_{0} & A_{\zeta}, \mathbb{X}_{0}, A & B_{0} & \sigma_{1} \\
\mathbb{C}^{2} & \mathcal{K} & \mathbb{C}^{2} &
\end{array}\right]
$$

where $A, A_{\zeta}$ are generators of analytic semi groups. The transfer function of the node $\mathfrak{N}_{0}$ is equal to $S(\lambda)$.

Proof: We are going to explicitly construct such a node, based on (37). Let us define two measures on column vector-functions with 2 entries:

$$
d \bar{\rho}^{+}=\left[\begin{array}{cc}
d \rho_{11}^{+}+d\left|\rho_{12}\right| & i d \rho_{12} \\
-i d \rho_{12} & d \rho_{22}^{+}+d\left|\rho_{12}\right|
\end{array}\right], \quad d \bar{\rho}^{-}=\left[\begin{array}{cc}
d \rho_{11}^{-}+d\left|\rho_{12}\right| & 0 \\
0 & d \rho_{22}^{-}+d\left|\rho_{12}\right|
\end{array}\right] .
$$

It is easy to see that $d \bar{\rho}=d \bar{\rho}^{+}-d \bar{\rho}^{-}$and we denote by $d|\bar{\rho}|=d \bar{\rho}^{+}+d \bar{\rho}^{-}$. Define a Hilbert space $\mathcal{H}$ of column vector-functions with 2 entries as follows $(\mathfrak{R}=\operatorname{supp}(d \bar{\rho}))$

$$
\mathcal{H}=\left\{\bar{u}(\mu)=\left[\begin{array}{l}
u_{1}(\mu) \\
u_{2}(\mu)
\end{array}\right] \in \mathbb{R}^{2}\left|\int_{\mathfrak{R}} \bar{u}^{*}(\mu) d\right| \bar{\rho}(\mu) \mid \bar{u}(\mu)<\infty\right\},
$$


equipped with the inner product

$$
\langle\bar{u}, \bar{v}\rangle_{\mathcal{H}}=\int_{\mathfrak{R}} \bar{v}^{*}(\mu) d|\bar{\rho}(\mu)| \bar{u}(\mu) .
$$

This is a well defined object, because the measures $d \bar{\rho}^{+}, d \bar{\rho}^{+}$are positive on the corresponding vector-functions. The positivity of $d \bar{\rho}^{-}$is immediate using (38) on arbitrary integrable $\left[\begin{array}{l}v_{1}(\mu) \\ v_{2}(\mu)\end{array}\right]$ :

$$
\begin{aligned}
\int_{\mathfrak{R}}\left[\begin{array}{ll}
v_{1}^{*}(\mu) & v_{2}^{*}(\mu)
\end{array}\right]\left[\begin{array}{cc}
d \rho_{11}^{-}+d\left|\rho_{12}\right| & 0 \\
0 & d \rho_{22}^{-}+d\left|\rho_{12}\right|
\end{array}\right]\left[\begin{array}{c}
v(\mu) \\
v(\mu)
\end{array}\right]= \\
\quad=\int_{\mathfrak{R}}\left|v_{1}\right|^{2}\left(d \rho_{11}^{-}+d\left|\rho_{12}\right|\right)+\int_{\mathfrak{R}}\left|v_{2}\right|^{2}\left(d \rho_{22}^{-}+d\left|\rho_{12}\right|\right) \geq 0 .
\end{aligned}
$$

For the measure $d \bar{\rho}^{+}$we need more computations and the following formula can be shown:

$$
\begin{aligned}
\int_{\Re}\left[\begin{array}{ll}
v_{1}^{*}(\mu) & v_{2}^{*}(\mu)
\end{array}\right]\left[\begin{array}{cc}
d \rho_{11}^{+}+d\left|\rho_{12}\right| & i d \rho_{12} \\
-i d \rho_{12} & d \rho_{22}^{+}+d\left|\rho_{12}\right|
\end{array}\right]\left[\begin{array}{c}
v(\mu) \\
v(\mu)
\end{array}\right]= \\
=\int_{\Re}\left|v_{1}\right|^{2} d \rho_{11}^{+}+\int_{\Re}\left|v_{2}\right|^{2} d \rho_{22}^{+}+\int_{\Re}\left(\Re v_{1}+\Im v_{2}\right)^{2} d \rho_{12}^{+}+\int_{\Re}\left(\Re v_{2}-\Im v_{1}\right)^{2} d \rho_{12}^{+} \geq 0 .
\end{aligned}
$$

We define a Krein space $\mathcal{K}=\mathcal{H}$ as a set, equipped with the following sesqui-linear form

$$
[\bar{u}, \bar{v}]_{\mathcal{K}}=\int_{\mathbb{R}} \bar{v}^{*}(\mu) d \bar{\rho}(\mu) \bar{u}(\mu)
$$

Define the operator $A=i \mu$ as the multiplication operator and

$$
A_{\zeta} f=-i \mu f(\mu)-\sigma_{1} \int_{\mathbb{R}} d \rho(\delta) f(\delta) .
$$

The operator $A_{\zeta}$ is a two-dimensional perturbation of the operator $-A$ : each function $f(\mu)$ is mapped by $A_{\zeta}$ to the sum of $-i \mu f(\mu)$ and a constant function $K=-\sigma_{1} \int_{\mathbb{R}} d \rho(\delta) f(\delta)$. The operators are generators of analytic semi-groups. Indeed, the group for $A$ is given by $e^{i \mu x}$ and is unitary. For the operator $A_{\zeta}$, we notice that for big enough $\lambda>0$, we can explicitly write the inverse of $\lambda I-A_{\zeta}$. From

$$
\left(\lambda I-A_{\zeta}\right) f=(\lambda+i \mu) f(\mu)+\sigma_{1} B_{0}^{*} f=(\lambda+i \mu) f(\mu)+\sigma_{1} K=g(\mu)
$$

it follows that

$$
\left(\lambda I-A_{\zeta}\right)^{-1} g=\frac{g(\mu)-\sigma_{1} K}{\lambda+i \mu},
$$

where the constant vector $K=K(\lambda, g)$ is found from the condition $K=B_{0}^{*}\left[\frac{g(\mu)-\sigma_{1} K}{\lambda+i \mu}\right]$. Solving it we find that

$$
\left[I+\sigma_{1} \int_{0}^{\infty} \frac{d \bar{\rho}(\mu)}{\lambda+i \mu}\right] K=\int_{0}^{\infty} \frac{d \bar{\rho}(\mu) g(\mu)}{\lambda+i \mu}
$$

and since for big enough $|\lambda|$ it holds that $\left\|\sigma_{1} \int_{0}^{\infty} \frac{d \bar{\rho}(\mu)}{\lambda+i \mu}\right\|<1$, we obtain

$$
K=\left[I+\sigma_{1} \int_{0}^{\infty} \frac{d \bar{\rho}(\mu)}{\lambda+i \mu}\right]^{-1} \int_{0}^{\infty} \frac{d \bar{\rho}(\mu) g(\mu)}{\lambda+i \mu} .
$$


From here it follows immediately that $\left(\lambda I-A_{\zeta}\right)^{-1}$ is bounded and $A_{\zeta}$ is a generator of an analytic semi group.

Obviously, $D\left(A_{\zeta}\right)=D(A)$. Define $\mathbb{X}_{0}=I: \mathcal{K} \rightarrow \mathcal{K}$ - the identity operator. So, the conditions $\mathbb{X}_{0}(D(A))=D(A)=\mathbb{X}_{0}^{-1}(D(A))$ of an invertible node are fulfilled. Define $B_{0}=C_{0}^{*}=I: \mathbb{C}^{2} \rightarrow \mathcal{K}$ and notice that $C_{0}: \mathcal{K} \rightarrow \mathbb{C}^{2}$ is an integration as follows $(f \in \mathcal{K})$

$$
C_{0} f u=\int_{\mathbb{R}} d \bar{\rho}(\mu) f(\mu)
$$

Then we compute for each $f \in D(A)$

$$
A \mathbb{X}_{0} f(\mu)+\mathbb{X}_{0} A_{\zeta} f(\mu)+B_{0} \sigma_{1} C_{0} f(\mu)=i \mu f(\mu)-i \mu f(\mu)-\sigma_{1} \int_{\mathbb{R}} d \rho(\delta) f(\delta)+\sigma_{1} \int_{\mathbb{R}} d \bar{\rho}(\mu) \bar{u}(\mu)=0,
$$

which means that the set

$$
\mathfrak{N}_{0}=\left[\begin{array}{cccc}
I^{*} & A_{\zeta}, I, A & I & \sigma_{1} \\
\mathbb{C}^{2} & \mathcal{K} & \mathbb{C}^{2} &
\end{array}\right]
$$

is an invertible node. Its transfer function is

$$
\begin{aligned}
I-C_{0} \mathbb{X}_{0}^{-1}(\lambda I-A)^{-1} B_{0} \sigma_{1} & =I-\int_{\mathbb{R}} I d \bar{\rho}(\mu) I(\lambda-i \mu)^{-1} I \sigma_{1} \\
& =I-\int_{\mathbb{R}} \frac{1}{\lambda-i \mu} d \bar{\rho}(\mu) \sigma_{1} \\
& =S(\lambda)
\end{aligned}
$$

and the Theorem follows.

\subsection{Construction of a vessel, realizing a given analytic potential}

Suppose that an analytic function $q(x)$ is given. We assume that $x_{0}=0$ for the simplicity of notations. Using results of the previous Section 4.1. or more precisely Corollary 4.4 we construct an invertible node $\mathfrak{N}_{0}$ to which we can apply the standard construction of a prevessel (see Section 3.2). We obtain in this manner a prevessel prev . Moreover, by Theorem 3.6 there exists an interval I, including $x_{0}$ and a vessel $\mathfrak{V}$ on I, such that the potential of the vessel $q_{V}(x)$ exists and is analytic. Moreover, from the form of the zero moment $H_{0}(x)$, by observing its 1,1 entry we will obtain that $q_{V}(x)=q(x)$ on I. So, if we are able to show that actually the vessel $\mathfrak{V}$ exists on the whole $\mathbb{R}$, we will realize the given potential by a vessel, constructing a scattering theory for it.

The following Theorem 4.6 shows that there exists a transfer function $S(\lambda, x)$, which realizes the given potential. This is a first sign that a vessel $\mathfrak{V}$ realizing $q(x)$ on $\mathbb{R}$ exists. Let us denote by $\Phi(\mu, x), \Phi_{*}(\mu, x)$ the fundamental solutions of (18), (19) respectively. First we notice that the columns and rows of the fundamental matrices are in $\mathcal{K}$. Indeed

$$
\Phi(i \mu, x)=\left[\begin{array}{cc}
\cos (\sqrt{\mu} x) & -i \sqrt{\mu} \sin (\sqrt{\mu} x) \\
-i \frac{\sin (\sqrt{\mu} x)}{\sqrt{\mu}} & \cos (\sqrt{\mu} x)
\end{array}\right]
$$

and it is obvious that $|\Phi(i \mu, x)|<\sqrt{\mu} C$ for some constant. Since $d|\rho(\mu)|$ is analytic the integral

$$
\int_{0}^{\infty}|\Phi(i \mu, x)|^{2} d|\rho(\mu)|<C^{2} \int_{0}^{\infty} \mu d|\rho(\mu)|
$$

is finite, which means that the columns and rows of $\Phi(i \mu, x)$ are in $\mathcal{K}$. To prove that the columns and rows of $\Phi_{*}(i \mu, x)$ are in $\mathcal{K}$ for each $x \in \mathbb{R}$, we need to learn its structure first. From (32), (33) it follows that

$$
\Phi_{*}(i \mu, x)=\left[\begin{array}{cc}
\phi(\mu, x) & i \psi(\mu, s) \\
-i\left(\frac{\partial}{\partial x} \phi(\mu, x)-\beta(x) \phi(\mu, x)\right) & \frac{\partial}{\partial x} \psi(\mu, x)-\beta(x) \psi(\mu, x)
\end{array}\right]
$$


where $\beta(x)=-\frac{1}{2} \int_{0}^{x} q(y) d y$ and $\phi(\mu, x), \psi(\mu, x)$ are solutions of (2) with the initial conditions

$$
\phi(\mu, 0)=1, \quad \frac{\partial}{\partial x} \phi(\mu, 0)=0, \quad \psi(\mu, 0)=0, \quad \frac{\partial}{\partial x} \psi(\mu, 0)=1 .
$$

The structure of these solutions is very well known Fad74]. Using variation of coefficients they satisfy

$$
\begin{aligned}
& \phi(\mu, x)=\cos (\sqrt{\mu} x)+\int_{0}^{x} \frac{\sin (\sqrt{\mu}(x-y))}{\sqrt{\mu}} q(y) \phi(\mu, y) d y, \\
& \psi(\mu, x)=\sin (\sqrt{\mu} x)+\int_{0}^{x} \frac{\sin (\sqrt{\mu}(x-y))}{\sqrt{\mu}} q(y) \psi(\mu, y) d y .
\end{aligned}
$$

And from their Liouville-Neumann series solutions we obtain

$$
\begin{aligned}
\phi(\mu, x)=\cos (\sqrt{\mu} x)- & \int_{0}^{x} \frac{\sin (\sqrt{\mu}(x-y))}{\sqrt{\mu}} q(y) \cos (\sqrt{\mu} y) d y+ \\
& +\int_{0}^{x} \frac{\sin (\sqrt{\mu}(x-y))}{\sqrt{\mu}} q(y) \int_{0}^{y} \frac{\sin \left(\sqrt{\mu}\left(y-y_{1}\right)\right)}{\sqrt{\mu}} q\left(y_{1}\right) \cos (\sqrt{\mu} y) d y_{1} d y-\cdots
\end{aligned}
$$

Since $\left|\frac{\sin (\sqrt{\mu}(x-y))}{\sqrt{\mu}}\right|,|\cos (\sqrt{\mu} y)|<1,|q(y)|<M_{x}$ on $[0, x]$ (or on $[x, 0]$ for $x<0$ ) for a constant $M_{x}>0$

$$
\sup _{\mathbb{R}}|\phi(\mu, x)|<1+\int_{0}^{|x|} M_{x} d y+\int_{0}^{|x|} \int_{0}^{y} M_{x}^{2} d y_{1} d y+\cdots=e^{M_{x}|x|}<\infty .
$$

Differentiating the formula for $\phi(\mu, x)$ we find that

$$
\frac{\partial}{\partial x} \phi(\mu, x)=-\sqrt{\mu} \sin (\sqrt{\mu} x)+\int_{0}^{x} \cos (\sqrt{\mu}(x-y)) q(y) \phi(\mu, y) d y
$$

from where it follows that $\left|\frac{\partial}{\partial x} \phi(\mu, x)\right|<C \sqrt{\mu}$, knowing the bound for $|\phi(\mu, x)|$. Similarly, one finds that $\phi(\mu, x)$ and its $x$-derivative satisfy the same bounds and as a result $\left|\Phi_{*}(\mu, x)\right|<\sqrt{\mu} C$ and the columns and rows of $\Phi_{*}(\mu, x)$ are in $\mathcal{K}$.

Theorem 4.6 (Transfer function construction). Let $q(x)$ be an analytic functions and let $H_{n}(x)$ be the moments, constructed in (35). Suppose that $S(\lambda)$ is realized in the form (37)

$$
S(\lambda)=I-\int_{\mathbb{R}} \frac{1}{\lambda-i \mu} d \bar{\rho}(\mu) \sigma_{1},
$$

with an analytic measure $d \bar{\rho}$ and satisfies $H_{n}^{S}=H_{n}(0)$. Let $\Phi(\lambda, x), \Phi_{*}(\lambda, x)$ be the fundamental solutions of (18), (19) respectively. Then the function

$$
S(\lambda, x)=I-\int_{\mathbb{R}} \frac{1}{\lambda-i \mu} \Phi_{*}(i \mu, x) d \bar{\rho}(\mu) \Phi^{*}(i \mu, x) \sigma_{1},
$$

satisfies $H_{n}^{S}(x)=H_{n}(x)$ for all $x \in \mathbb{R}$. 
Proof: Differentiating the $n$-th moment of $S(\lambda, x)$

$$
H_{n}^{S}(x)=\int_{\mathbb{R}} \Phi_{*}(i \mu, x) d \bar{\rho}(\mu)(i \mu)^{n} \Phi^{*}(i \mu, x)
$$

we find that

$$
\begin{gathered}
\frac{\partial}{\partial x} H_{n}^{S}(x)=\int_{\mathbb{R}} \sigma_{1}^{-1}\left(\sigma_{2} i \mu+\gamma_{*}(x)\right) \Phi_{*}(i \mu, x) d \bar{\rho}(\mu)(i \mu)^{n} \Phi^{*}(i \mu, x)- \\
-\int_{\mathbb{R}} \Phi_{*}(i \mu, x) d \bar{\rho}(\mu)(i \mu)^{n} \Phi^{*}(i \mu, x)\left(\sigma_{2} i \mu+\gamma\right) \sigma_{1}^{-1}= \\
=\sigma_{1}^{-1} \sigma_{2} H_{n+1}^{S}-H_{n+1}^{S} \sigma_{2} \sigma_{1}^{-1}+\sigma_{1}^{-1} \gamma_{*} H_{n}^{S}-H_{n}^{S} \gamma \sigma_{1}^{-1}
\end{gathered}
$$

which is identical to (31). So $H_{n}^{S}(x)$ and $H_{n}(x)$ have the same initial conditions and satisfy the same differential equations, so they are identical by the uniqueness of the moments Theorem 3.10.

Corollary 4.7. $H_{n}(x)=\int_{\mathbb{R}} \Phi_{*}(i \mu, x) d \bar{\rho}(\mu)(i \mu)^{n} \Phi^{*}(i \mu, x)$. Particularly, for $n=0$

$$
H_{0}(x)=\int_{\mathbb{R}} \Phi_{*}(i \mu, x) d \bar{\rho}(\mu) \Phi^{*}(i \mu, x) .
$$

Assume that for a given analytic $q(x)$ we have constructed moments $H_{n}(x)$, a measure $d \bar{\rho}$ in Corollary 4.4 and a node $\mathfrak{N}_{0}$ (Theorem 4.5). Applying the standard construction Theorem 3.5 to $\mathfrak{N}_{0}$, we obtain a prevessel:

$$
\mathfrak{p r e V}=\left[\begin{array}{cccc}
C(x) & A_{\zeta}, \mathbb{X}(x), A & B(x) & \sigma_{1}, \sigma_{2}, \gamma \\
\mathbb{C}^{2} & \mathcal{K} & \mathbb{C}^{2} &
\end{array}\right] .
$$

In order to show that the operator $\mathbb{X}(x)$ is globally defined, we construct an "inverse vessel" as follows. We use similar to (24), (25) definitions, using the fundamental matrix $\Phi_{*}(\lambda, x)$ instead of $\Phi(\lambda, x)$ :

Definition 4.3. Define the operators

$$
\begin{array}{r}
B_{*}(x)=\frac{1}{2 \pi i} \int_{\Gamma}\left(\lambda I+A_{\zeta}\right)^{-1} B_{0} \Phi_{*}^{*}(-\bar{\lambda}, x) d \lambda, \\
C_{*}(x)=\frac{1}{2 \pi i} \int_{\Gamma} \Phi_{*}(\lambda, x) C_{0}(\lambda I-A)^{-1} d \lambda, \\
\mathbb{X}_{*}(x)=I-\int_{0}^{x} B_{*}(x) \sigma_{2} C_{*}(y) d y .
\end{array}
$$

Lemma 4.8. The operators $B_{*}(x), C_{*}(x), \mathbb{X}_{*}(x)$ satisfy

$$
\begin{array}{r}
\frac{d}{d x} B_{*}(x)=\left(A_{\zeta} B_{*}(x) \sigma_{2}-B_{*}(x) \gamma_{*}(x)\right) \sigma_{1}^{-1}, \quad B_{*}(0)=B_{0}, \\
\frac{d}{d x} C_{*}(x) u=\sigma_{1}^{-1}\left(\sigma_{2} C(x) A+\gamma_{*}(x) C_{*}(x)\right) u, \quad u \in D(A), \quad C_{*}(0)=C_{0}, \\
A_{\zeta} \mathbb{X}_{*}(x) u+\mathbb{X}_{*}(x) A u+B_{*}(x) \sigma_{1} C_{*}(x) u=0, \quad u \in D(A) .
\end{array}
$$

Proof: Immediate from the definitions. The Lyapunov equation (48) follows similarly to the proof of Lemma 3.2 
Lemma 4.9. Define moments $G_{n}(x)=C_{*}(x) A^{n} B(x)$. Then $G_{n}(x)=H_{n}(x)$, particularly

$$
C_{*}(x) B(x)=H_{0}(x) .
$$

Proof: The moments $G_{n}(x)$ are well defined, since $A^{n} B(x)$ is an element of $\mathcal{K}$ for each $n$. Then

$$
\begin{aligned}
& \frac{d}{d x} G_{n}(x)=\frac{d}{d x}\left[C_{*}(x) A^{n} B(x)\right]= \\
& =\sigma_{1}^{-1}\left(\sigma_{2} C_{*}(x) A^{n+1}+\gamma_{*}(x) C_{*}(x) A^{n}\right) B(x)-C_{*}(x) A^{n}\left(A B(x) \sigma_{2}+B(x) \gamma\right) \sigma_{1}^{-1}= \\
& =\sigma_{1}^{-1}\left(\sigma_{2} G_{n+1}(x)+\gamma_{*}(x) G_{n}(x)\right)-\left(G_{n+1}(x) \sigma_{2}+G_{n}(x) \gamma\right)^{-1}= \\
& \quad=\sigma_{1}^{-1} \sigma_{2} G_{n+1}(x)-G_{n+1}(x) \sigma_{2} \sigma_{1}^{-1}+\sigma_{1}^{-1} \gamma_{*}(x) G_{n}(x)-G_{n}(x) \gamma \sigma_{1}^{-1},
\end{aligned}
$$

which coincides with (31). Moreover, $G_{n}(0)=H_{n}(0)=C_{0} A^{n} B_{0}$ by their constructions. So, by the uniqueness of the moments Theorem 3.10 $G_{n}(x)=H_{n}(x)$.

From the equation (49) we obtain relations between the operators:

Theorem 4.10. The following formulas hold

$$
\begin{gathered}
\mathbb{X}(x) B_{*}(x)=B(x), \quad C_{*}(x) \mathbb{X}(x)=C(x), \\
\mathbb{X}_{*}(x) B(x)=B_{*}(x), \quad C(x) \mathbb{X}_{*}(x)=C_{*}(x) .
\end{gathered}
$$

Proof: Let us prove the identity $C_{*}(x) \mathbb{X}(x)=C(x)$ and the rest are obtained in a similar manner. The following identities are applied to an element $u \in D(A)$ :

$$
\begin{aligned}
\frac{d}{d x}\left[C_{*}(x) \mathbb{X}(x)\right]= & \sigma_{1}^{-1}\left[\sigma_{2} C_{*}(x) A+\gamma_{*}(x) C_{*}(x)\right] \mathbb{X}(x)+C_{*}(x) B(x) \sigma_{2} C(x) \\
= & \sigma_{1}^{-1} \sigma_{2} C_{*}(x) A \mathbb{X}(x)+\gamma_{*}(x) C_{*}(x) \mathbb{X}(x)+H_{0}(x) \sigma_{2} C(x) \\
= & \operatorname{using}(\underline{6}) \\
= & \sigma_{1}^{-1} \sigma_{2} C_{*}(x)\left[-\mathbb{X}(x) A_{\zeta}-B(x) \sigma_{1} C(x)\right]+\sigma_{1}^{-1} \gamma_{*}(x) C_{*}(x) \mathbb{X}(x)+H_{0}(x) \sigma_{2} C(x) \\
= & -\sigma_{1}^{-1} \sigma_{2} C_{*}(x) \mathbb{X}(x) A_{\zeta}-\sigma_{1}^{-1} \sigma_{2} C_{*}(x) B(x) \sigma_{1} C(x)+ \\
& \quad+\sigma_{1}^{-1} \gamma_{*}(x) C_{*}(x) \mathbb{X}(x)+H_{0}(x) \sigma_{2} C(x) \\
= & \operatorname{using}(49) \text { and (14) } \\
= & -\sigma_{1}^{-1} \sigma_{2}\left[C_{*}(x) \mathbb{X}(x)\right] A_{\zeta}+\sigma_{1}^{-1} \gamma_{*}(x)\left[C_{*}(x) \mathbb{X}(x)\right]-\left[\gamma_{*}(x)-\gamma\right] C(x)
\end{aligned}
$$

in other words the operator $C_{*}(x) \mathbb{X}(x)$ satisfies the following non-homogeneous differential equation

$$
\frac{d}{d x} Y=-\sigma_{1}^{-1} \sigma_{2} Y A_{\zeta}+\sigma_{1}^{-1} \gamma_{*}(x) Y-\left[\gamma_{*}(x)-\gamma\right] C(x)
$$

On the other hand, $C(x)$ satisfies the same differential equation:

$$
\frac{d}{d x} C(x)=-\sigma_{1}^{-1} \sigma_{2} C(x) A_{\zeta}+\sigma_{1}^{-1} \gamma_{*}(x) C(x)-\left[\gamma_{*}(x)-\gamma\right] C(x)=\sigma_{1}^{-1}\left(-\sigma_{2} C(x) A_{\zeta}+\gamma C(x)\right) .
$$

Since $C_{*}(0) \mathbb{X}(0)=C(0)=C_{0}$ the result follows, by the uniqueness of the solution. Similarly, using (10), 45) and (48)

$$
\frac{d}{d x}\left[\mathbb{X}_{*}(x) B(x)\right]=A_{\zeta}\left[\mathbb{X}_{*}(x) B(x)\right] \sigma_{2} \sigma_{1}^{-1}-\left[\mathbb{X}_{*}(x) B(x)\right] \gamma \sigma_{1}^{-1}-B_{*}(x)\left[\gamma_{*}(x)-\gamma\right] \sigma_{1}^{-1},
$$

and $\mathbb{X}_{*}(x) B(x)$, substituted with $B_{*}(x)$ satisfies the same differential equation. Thus $\mathbb{X}_{*}(x) B(x)=$ $B_{*}(x)$. As a result, we obtain that

$$
C(x) B_{*}(x)=C(x) \mathbb{X}_{*}(x) B(x)=C_{*}(x) B(x)=H_{0}(x) .
$$

Then the equations $C(x) \mathbb{X}_{*}(x)=C_{*}(x), \mathbb{X}(x) B_{*}(x)=B(x)$ follows in the same manner. 
Corollary 4.11. The operator $\mathbb{X}(x)$ is invertible for all $x \in \mathbb{R}$ with the inverse $\mathbb{X}_{*}(x)$.

Proof: Notice that from Theorem 4.10 it follows that

$$
\begin{gathered}
\frac{d}{d x}\left[\mathbb{X}(x) \mathbb{X}_{*}(x)\right]=B(x) \sigma_{2} C(x) \mathbb{X}_{*}(x)-\mathbb{X}(x) B_{*}(x) \sigma_{2} C_{*}(x)=B(x) \sigma_{2} C_{*}(x)-B(x) \sigma_{2} C_{*}(x)=0, \\
\frac{d}{d x}\left[\mathbb{X}_{*}(x) \mathbb{X}(x)\right]=-B_{*}(x) \sigma_{2} C_{*}(x) \mathbb{X}(x)-\mathbb{X}_{*}(x) B(x) \sigma_{2} C(x)=B_{*}(x) \sigma_{2} C(x)-B_{*}(x) \sigma_{2} C(x)=0 .
\end{gathered}
$$

Since $\mathbb{X}(0) \mathbb{X}_{*}(0)=\mathbb{X}_{*}(0) \mathbb{X}(0)=I$, the Corollary follows.

Theorem 4.12. The vessel,

$$
\mathfrak{V}=\left[\begin{array}{cccc}
C(x) & A_{\zeta}, \mathbb{X}(x), A & B(x) & \sigma_{1}, \sigma_{2}, \gamma, \gamma_{*}(x) \\
\mathbb{C}^{2} & \mathcal{K} & \mathbb{C}^{2} & \mathbb{R}
\end{array}\right]
$$

obtained by applying the standard construction to the node $\mathfrak{N}_{0}$ exists on $\mathbb{R}$. The matrix function $\gamma_{*}(x)$, defined by the linkage condition (14) realizes the potential $q(x)$ on $\mathbb{R}$.

Proof: Since $\mathbb{X}(x)$ is globally invertible, we can repeat the proof of Theorem 3.6] in order to show that the prevessel prev is an invertible node. this shows that $\mathfrak{V}$ is a vessel, realizing an analytic potential $q_{V}(x)$ on $\mathbb{R}$, since $\mathbb{X}(x)$ is globally invertible. Then by theorem 4.6 the moments of the vessel $\mathfrak{V}$ are equal to the moments $H_{n}(x)$, particularly $H_{0}(x)=C(x) \mathbb{X}^{-1}(x) B(x)$ for which the 1,1 entry means that $q_{V}(x)=q(x)$.

\section{$5 \mathrm{KdV}$ evolutionary vessels}

Let us evolve a SL vessel with respect to $t$. Some of the results presented here can be found in Melf Melc for symmetric vessels.

We consider the following notion

Definition 5.1. The collection of operators and spaces

$$
\mathfrak{p r e} \mathfrak{V}_{K d V}=\left[\begin{array}{cccc}
C(x, t) & A_{\zeta}, \mathbb{X}(x, t), A & B(x, t) & \sigma_{1}, \sigma_{2}, \gamma \\
\mathbb{C}^{2} & \mathcal{K} & \mathbb{C}^{2}
\end{array}\right]
$$

is called a KdV pre Vessel, if the following conditions hold: 1. pre $\mathfrak{V}_{K d V}$ is a node for all $x, t \in \mathbb{R}, 2$. operator $B(x, t) \sigma_{2}$ is $A^{2}$-regular, $B(x, t) \gamma$ is $A$-regular 3. $C(x, t), \mathbb{X}(x, t), B(x, t)$ are differentiable in both variables, when the other one is fixed, subject to the conditions (10), (11), (12) and the following evolutionary equations (for arbitrary $u \in D(A), v \in D(A)$ )

$$
\begin{array}{ccc}
\frac{\partial}{\partial t} B & =i A \frac{\partial}{\partial x} B & =-i A\left(A B \sigma_{2}+B \gamma\right) \sigma_{1}^{-1}, \\
\frac{\partial}{\partial t} C u & =-i \frac{\partial}{\partial x} C A_{\zeta} u & =-i \sigma_{1}^{-1}\left(-\sigma_{2} C A_{\zeta}+\gamma C\right) A_{\zeta} u, \\
\frac{\partial}{\partial t} \mathbb{X} v=i\left(A \frac{\partial}{\partial x} \mathbb{X}-i \frac{\partial}{\partial x} \mathbb{X} A_{\zeta}+i B \gamma C\right) v & =i\left(A B \sigma_{2} C-i B \sigma_{2} C A_{\zeta}+i B \gamma C\right) v,
\end{array}
$$

where $\sigma_{2}=\sigma_{2}^{*}, \gamma^{*}=-\gamma$ are $2 \times 2$ matrices. The prevessel prev is called symmetric if $A_{\zeta}=A^{*}$ and $C(x, t)=B^{*}(x, t)$ for all $x, t \in \mathbb{R}$.

Definition 5.2. The collection of operators, spaces and an open set $\Omega \subseteq \mathbb{R}^{2}$

$$
\mathfrak{V}_{K d V}=\left[\begin{array}{cccc}
C(x, t) & A_{\zeta}, \mathbb{X}(x, t), A & B(x, t) & \sigma_{1}, \sigma_{2}, \gamma, \gamma_{*}(x, t) \\
\mathbb{C}^{2} & \mathcal{K} & \mathbb{C}^{2} & \Omega
\end{array}\right]
$$

is called a (non-symmetric) $\boldsymbol{K} \boldsymbol{d} \boldsymbol{V}$ vessel, if $\mathfrak{V}_{K d V}$ is a KdV prevessel, $\mathbb{X}(x, t)$ is invertible on $\Omega$, $\mathfrak{V}_{K d V}$ is also an invertible node. The $2 \times 2$ matrix-function $\gamma_{*}(x, t)$ satisfies the linkage condition (14). The vessel $\mathfrak{V}_{K d V}$ is called symmetric if $A_{\zeta}=A^{*}$ and $C(x, t)=B^{*}(x, t)$ for all $x, t \in \Omega$. 
Theorem 5.1. Let $\mathfrak{V}_{K d V}$ be a KdV vessel. Suppose that the moments $H_{0}, \ldots, H_{n+1}$ are finite and differentiable, then

$$
\frac{\partial}{\partial t} H_{n}=i \frac{\partial}{\partial x} H_{n+1}+i \frac{\partial}{\partial x}\left[H_{0}\right] \sigma_{1} H_{n}
$$

The transfer function $S(\lambda, x, t)$ (7) satisfies the following differential equation

$$
\frac{\partial}{\partial t} S(\lambda, x, t)=i \lambda \frac{\partial}{\partial x} S(\lambda, x, t)+i \frac{\partial}{\partial x}\left[H_{0}\right] \sigma_{1} S(\lambda, x, t) .
$$

Proof: Consider the formula for the moments first.

$$
\begin{aligned}
\frac{\partial}{\partial t} H_{n} & =\frac{\partial}{\partial t}\left[C \mathbb{X}^{-1} A^{n} B\right]=C_{t} \mathbb{X}^{-1} A^{n} B-C \mathbb{X}^{-1} \mathbb{X}_{t} \mathbb{X}^{-1} A^{n} B+C \mathbb{X}^{-1} A^{n} B_{t}= \\
& =\text { using evolutionary conditions (51) },(\underline{(53)} \\
& =C_{x}\left(-i A_{\zeta}\right) \mathbb{X}^{-1} A^{n} B-C \mathbb{X}^{-1}\left(i A \mathbb{X}_{x}-i \mathbb{X}_{x} A_{\zeta}+i B \gamma C\right) \mathbb{X}^{-1} A^{n} B+C \mathbb{X}^{-1} A^{n}(i A) B_{x}= \\
& =u \operatorname{sing}(\mathbb{1 0}),(14) \text { and (6) } \\
& =i \frac{\partial}{\partial x} H_{n+1}+i \frac{\partial}{\partial x}\left[H_{0}\right] \sigma_{1} H_{n},
\end{aligned}
$$

Similarly one shows the formula (56).

Corollary 5.2. The potential $\gamma_{*}(x, t)$ of a KdV vessel satisfies the following differential equation

$$
\left(\gamma_{*}\right)_{t}=-i \gamma_{*}\left(H_{0}\right)_{x} \sigma_{1}+i \sigma_{1}\left(H_{0}\right)_{x x} \sigma_{1}+i \sigma_{1}\left(H_{0}\right)_{x} \gamma_{*} .
$$

Proof: From the linkage condition and (55) for $n=0$ it follows that

$$
\begin{aligned}
\left(\gamma_{*}\right)_{t} & =\sigma_{2}\left(H_{0}\right)_{t} \sigma_{1}-\sigma_{1}\left(H_{0}\right)_{t} \sigma_{2}= \\
& =\sigma_{2}\left[i\left(H_{1}\right)_{x}+i\left(H_{0}\right)_{x} \sigma_{1} H_{0}\right] \sigma_{1}-\sigma_{1}\left[i\left(H_{1}\right)_{x}+i\left(H_{0}\right)_{x} \sigma_{1} H_{0}\right] \sigma_{2} \\
& =i \sigma_{1}\left[\sigma_{1}^{-1} \sigma_{2}\left(H_{1}\right)_{x}-\left(H_{1}\right)_{x} \sigma_{2} \sigma_{1}^{-1}\right] \sigma_{1}+i \sigma_{2}\left(H_{0}\right)_{x} \sigma_{1} H_{0} \sigma_{1}-i \sigma_{1}\left(H_{0}\right)_{x} \sigma_{1} H_{0} \sigma_{2} .
\end{aligned}
$$

For the first term in this expression we can use the formula (31) for $n=0$, then

$$
\begin{aligned}
\left(\gamma_{*}\right)_{t} & =i \sigma_{1} \frac{\partial}{\partial x}\left[\left(H_{0}\right)_{x}-\sigma_{1}^{-1} \gamma_{*} H_{0}+H_{0} \gamma \sigma_{1}^{-1}\right] \sigma_{1}+i \sigma_{2}\left(H_{0}\right)_{x} \sigma_{1} H_{0} \sigma_{1}-i \sigma_{1}\left(H_{0}\right)_{x} \sigma_{1} H_{0} \sigma_{2} \\
& =i \sigma_{1}\left(H_{0}\right)_{x x} \sigma_{1}-i \frac{\partial}{\partial x}\left[\gamma_{*} H_{0} \sigma_{1}+\sigma_{1} H_{0} \gamma\right]+i \sigma_{2}\left(H_{0}\right)_{x} \sigma_{1} H_{0} \sigma_{1}-i \sigma_{1}\left(H_{0}\right)_{x} \sigma_{1} H_{0} \sigma_{2} \\
& =i \sigma_{1}\left(H_{0}\right)_{x x} \sigma_{1}-i \gamma_{*}\left(H_{0}\right)_{x} \sigma_{1}-i\left(\gamma_{*}\right)_{x} H_{0} \sigma_{1}+\sigma_{1}\left(H_{0}\right)_{x} \gamma+i \sigma_{2}\left(H_{0}\right)_{x} \sigma_{1} H_{0} \sigma_{1}-i \sigma_{1}\left(H_{0}\right)_{x} \sigma_{1} H_{0} \sigma_{2} .
\end{aligned}
$$

Then notice that

$$
\begin{aligned}
& -i\left(\gamma_{*}\right)_{x} H_{0} \sigma_{1}+\sigma_{1}\left(H_{0}\right)_{x} \gamma+i \sigma_{2}\left(H_{0}\right)_{x} \sigma_{1} H_{0} \sigma_{1}-i \sigma_{1}\left(H_{0}\right)_{x} \sigma_{1} H_{0} \sigma_{2}= \\
& =-i\left[\sigma_{2}\left(H_{0}\right)_{x} \sigma_{1}-\sigma_{1}\left(H_{0}\right)_{x} \sigma_{2}\right] H_{0} \sigma_{1}+\sigma_{1}\left(H_{0}\right)_{x} \gamma+i \sigma_{2}\left(H_{0}\right)_{x} \sigma_{1} H_{0} \sigma_{1}-i \sigma_{1}\left(H_{0}\right)_{x} \sigma_{1} H_{0} \sigma_{2}= \\
& =i \sigma_{1}\left(H_{0}\right)_{x}\left[\gamma+\sigma_{2} H_{0} \sigma_{1}-\sigma_{1} H_{0} \sigma_{2}\right] \\
& =i \sigma_{1}\left(H_{0}\right)_{x} \gamma_{*},
\end{aligned}
$$

and the result follows.

Corollary 5.3. The potential $q(x, t)$ of a $K d V$ vessel satisfies the $K d V$ equation (1) on $\Omega$, which is equivalent to (57).

Proof: From the linkage condition it follows that for $\beta=\frac{1}{2} \int_{0}^{x} q(y, t) d y$

$$
\gamma_{*}=\left[\begin{array}{cc}
-i\left(\beta_{x}^{\prime}-\beta^{2}\right) & -\beta \\
\beta & i
\end{array}\right] .
$$

Moreover, by (33), the $\mathrm{KdV}$ equation for $q(x, t)$ follows from the differential equation for $\beta(t, x)$ :

$$
-4 \beta_{t}=-6\left(\beta_{x}\right)^{2}+\beta_{x x x}
$$


Then using (57) and (14)

$$
\begin{aligned}
-\beta_{t} & =\left[\begin{array}{ll}
1 & 0
\end{array}\right]\left(\gamma_{*}\right)_{t}\left[\begin{array}{l}
0 \\
1
\end{array}\right]=\left[\begin{array}{ll}
1 & 0
\end{array}\right]\left[-i \gamma_{*}\left(H_{0}\right)_{x} \sigma_{1}+i \sigma_{1}\left(H_{0}\right)_{x x} \sigma_{1}+i \sigma_{1}\left(H_{0}\right)_{x} \gamma_{*}\right]\left[\begin{array}{l}
0 \\
1
\end{array}\right]= \\
& =\left[\begin{array}{ll}
1 & 0
\end{array}\right]\left[-i\left(\gamma+\sigma_{2} H_{0} \sigma_{1}-\sigma_{1} H_{0} \sigma_{2}\right)\left(H_{0}\right)_{x} \sigma_{1}+i \sigma_{1}\left(H_{0}\right)_{x x} \sigma_{1}+i \sigma_{1}\left(H_{0}\right)_{x} \gamma_{*}\right]\left[\begin{array}{l}
0 \\
1
\end{array}\right] .
\end{aligned}
$$

We have seen in (34) that

$$
H_{0}=\left[\begin{array}{cc}
-\beta & \frac{-i \pi_{11}}{2} \\
\frac{i \pi_{11}}{2} & h_{0}^{22}
\end{array}\right]
$$

Plugging this formula into the last expression we will find that

$$
-\beta_{t}=-\left(h_{0}^{22}\right)_{x}-\beta_{x} \beta^{2}+2\left(\beta_{x}\right)^{2}+\beta \beta_{x x}-\frac{1}{2} \beta_{x x x} .
$$

From the last formula of (35) it follows that

$$
\left(h_{0}^{22}\right)_{x}=\frac{1}{2 i}\left[\left(\frac{-i \pi_{11}}{2}\right)_{x x}-2 \beta\left(\frac{-i \pi_{11}}{2}\right)_{x}\right]=-\beta^{2} \beta_{x}+\frac{1}{2}\left(\beta_{x}\right)^{2}+\beta \beta_{x x}-\frac{1}{4} \beta_{x x x} .
$$

Plugging this expression into the formula for $-\beta_{t}$, we will obtain (58)

$$
-\beta_{t}=\frac{3}{2}\left(\beta_{x}\right)^{2}-\frac{1}{4} \beta_{x x x}
$$

which the $\mathrm{KdV}$ equation for $\beta(x)$. Differentiating it with respect to $x$, we will obtain the regular $\mathrm{KdV}$ equation (1) for $q(x, t)=2 \beta_{x}(x, t)$. It is a matter of simple algebraic calculations to verify that the 1,1 entry of (57) is equivalent to (1), since the 1,1 entry of $\gamma_{*}(x, t)=-i\left(\beta_{x}-\beta^{2}\right)$ is expressible in terms of $\beta$.

Now we obtain the Main Theorem, because the fact that $\mathbb{X}(x)$ is invertible for a fixed $x$ implies that its norm is bounded from below and a small perturbation of it is still invertible.

Main Theorem 5.4. Suppose that $q(x)$ is an analytic function on $\mathbb{R}$. There exists a KdV vessel, which exists on $\Omega \subseteq \mathbb{R}^{2}$. For each $x \in \mathbb{R}$ there exists $T_{x}>0$ such that $\{x\} \times\left[-T_{x}, T_{x}\right] \in \Omega$. The potential $q(x)$ is realized by the vessel for $t=0$.

Proof: For the given analytic potential $q(x)$ we construct a SL vessel

$$
\begin{aligned}
& \mathfrak{V}=\left[\begin{array}{cccc}
C(x) & A_{\zeta}, \mathbb{X}(x), A & B(x) & \sigma_{1}, \sigma_{2}, \gamma, \gamma_{*}(x) \\
\mathbb{C}^{2} & \mathcal{K} & \mathbb{C}^{2} &
\end{array}\right] \\
& B(x)=\Phi^{*}(i \mu, x), \quad C(x)=\frac{1}{2 \pi i} \int_{\Gamma} \Phi(\lambda, x) C_{0}\left(\lambda I+A_{\zeta}\right)^{-1} d \lambda \\
& \mathbb{X}(x)=I+\int_{0^{*}}^{x} B(y) \sigma_{2} C(y) d y= \\
& =I+\int_{\Gamma} \frac{\Phi^{*}(i \mu, x) \sigma_{1} \Phi(\lambda, x)-\sigma_{1}}{(\lambda-i \mu)} C_{0}\left(\lambda I+A_{\zeta}\right)^{-1} d \lambda,
\end{aligned}
$$

defined in Theorem 4.12. The last formula for $\mathbb{X}(x)$ comes from an easily checkable fact that $\frac{\partial}{\partial x} \frac{\Phi^{*}(i \mu, x) \sigma_{1} \Phi(\lambda, x)-\sigma_{1}}{(\lambda-i \mu)}=\Phi^{*}(i \mu, x) \sigma_{2} \Phi(\lambda, x)$. On its basis we define A KdV vessel (54)

$$
\mathfrak{V}_{K d V}=\left[\begin{array}{cccc}
C(x, t) & A_{\zeta}, \mathbb{X}(x, t), A & B(x, t) & \sigma_{1}, \sigma_{2}, \gamma, \gamma_{*}(x, t) \\
\mathbb{C}^{2} & \mathcal{K} & \mathbb{C}^{2} & \Omega
\end{array}\right]
$$


as follows:

$$
\begin{aligned}
& B(x, t)=\Phi^{*}(i \mu, x-\mu t), \quad C(x, t)=\frac{1}{2 \pi i} \int_{\Gamma_{\Gamma}} \Phi(\lambda, x-i \lambda t) C_{0}\left(\lambda I+A_{\zeta}\right)^{-1} d \lambda, \\
& \mathbb{X}(x, t)=I+\int_{\Gamma} \frac{\Phi^{*}(i \mu, x-\mu t) \sigma_{1} \Phi(\lambda, x-i \lambda t)-\sigma_{1}}{(\lambda-i \mu)} C_{0}\left(\lambda I+A_{\zeta}\right)^{-1} d \lambda .
\end{aligned}
$$

It is a matter of simple algebraic calculations to verify that $B(x, t), C(x, t), \mathbb{X}(x, t)$ satisfy the conditions of a KdV prevessel. One has to use the fact that

$$
\begin{aligned}
\frac{\partial}{\partial t} \frac{\Phi^{*}(i \mu, x-\mu t) \sigma_{1} \Phi(\lambda, x-i \lambda t)-\sigma_{1}}{(\lambda-i \mu)} & = \\
& =i \Phi^{*}(i \mu, x-\mu t) \sigma_{2} \Phi(\lambda, x-i \lambda t)(\lambda+i \mu)+i \Phi^{*}(i \mu, x-\mu t) \gamma \Phi(\lambda, x-i \lambda t) .
\end{aligned}
$$

Finally, notice that for $t=0$ the operator $\mathbb{X}(x, 0)$ equals to the operator $\mathbb{X}(x)$ of the SL vessel, constructed for $q(x)$. Thus the set $\Omega$, on which $\mathbb{X}(x, t)$ is invertible includes $\mathbb{R} \times\{0\}$. Moreover, since $\left(A_{\zeta}^{*}\right)^{n} C^{*}(x) \sigma_{2}$ is exists for all $n$ by the construction, we obtain that $\sigma_{2} C(x) A_{\zeta}$ is a well defined BOUNDED functional on $\mathcal{K}$. Thus the expression

$$
A B(x, s) \sigma_{2} C(x, s)-i B(x, s)\left[\begin{array}{l}
1 \\
0
\end{array}\right]\left[A_{\zeta}^{*} C^{*}(x)\left[\begin{array}{l}
1 \\
0
\end{array}\right]\right]^{*}+i B(x, s) \gamma C(x, s)
$$

is a bounded operator on $\mathcal{K}$. As a result, the operator $\mathbb{X}(x, t)$ is bounded for some $t \in\left[-T_{x}, T_{x}\right]$, where

$$
0<T_{x}<\frac{\left\|A B(x, s) \sigma_{2} C(x, s)-i B(x, s) \sigma_{2} C(x, s) A_{\zeta}+i B(x, s) \gamma C(x, s)\right\|}{\left\|\mathbb{X}^{-1}(x, 0)\right\|} .
$$

Finally, we present a Theorem, providing a conclusion that this theory of vessels is the ultimate tool for studying solutions of (1). A most general Theorem in this connection is to show that if there is an open set $\Omega$, where solution is known to exist, then the operator $\mathbb{X}(x, t)$ is invertible in this region. Since we use the uniqueness of solutions for ODEs, the set $\Omega$ must be at least simply-connected. On the other hand, we do not want enter into topological difficulties, arising from such a general assumption, so we choose a very important and practical case of a strip. So, if it is known that the solution of (1) exists on $\mathbb{R} \times[0, T]$, we would like to show that the vessel, which realizes $q(x)$ at $t=0$ will exist on this strip.

The idea of the proof of such a Theorem is very simple. We actually explicitly construct the inevsrse of $\mathbb{X}(x, t)$, using the assumption that that there exists a solution $q(x, t)$ of $\mathbb{1}$ on $\mathbb{R} \times[0, T]$. For a potential $q(x, t)$ on the strip, which solves (1) we can define

$$
\beta(x, t)=\frac{1}{2} \int_{0}^{x} q(y, t) d y, \quad \pi_{11}(x, t)=\beta_{x}(x, t)-\beta^{2}(x, t), \quad \gamma_{*}(x, t) \text { by (14). }
$$

Deine also

$$
\frac{\partial}{\partial x} H_{0}(x, t)=\frac{\partial}{\partial x}\left[\begin{array}{cc}
-\beta(x, t) & -i \frac{\pi_{11}(x, t)}{2} \\
i \frac{\pi_{11}(x, t)}{2} & h_{0}^{22}(x, t)
\end{array}\right]
$$

where $\frac{\partial}{\partial x} h_{0}^{22}(x, t)$ is real-valued and satisfies the last equation of (35):

$$
2 i \frac{\partial}{\partial x} h_{0}^{22}(x, t)=-i \frac{\partial^{2}}{\partial x^{2}} \frac{\pi_{11}(x, t)}{2}+2 i \beta(x, t) \frac{\partial}{\partial x} \frac{\pi_{11}(x, t)}{2} .
$$


Then there exists a unique solution $\Phi_{*}(\lambda, x, t)$, satisfying the following system of equations

$$
\left\{\begin{array}{l}
\lambda \sigma_{2} \Phi_{*}(\lambda, x, t)-\sigma_{1} \frac{\partial}{\partial x} \Phi_{*}(\lambda, x, t)+\gamma_{*}(x, t) \Phi_{*}(\lambda, x, t)=0 \\
\frac{\partial}{\partial t} \Phi_{*}(\lambda, x, t)=i \lambda \frac{\partial}{\partial x} \Phi_{*}(\lambda, x, t)+i \frac{\partial}{\partial x}\left[H_{0}(x, t)\right] \sigma_{1} \Phi_{*}(\lambda, x, t) \\
\Phi_{*}(\lambda, 0,0)=I
\end{array}\right.
$$

Notice that for $t=0$ the fundamental matrix $\Phi_{*}(\lambda, x, 0)$ coincides with the fundamental matrix $\Phi_{*}(\lambda, x)$ considered earlier as the solution of (19). Evolving this solution using $t$-derivative, we will get the fundamental solution of (59). Notice that the system of equations (59) is uniquely solvable in $\mathbb{R} \times[0, T]$. The identity of second mixed partial derivatives

$$
\frac{\partial^{2}}{\partial x \partial t} \Phi_{*}(\lambda, x, t)=\frac{\partial^{2}}{\partial t \partial x} \Phi_{*}(\lambda, x, t)
$$

follows from (57), which is identical to (10) due to Corollary 5.3. We define the moments, associated with $q(x, t)$ as follows

$$
H_{n}(x, t)=\int_{0}^{\infty} \Phi_{*}(i \mu, x, t) d \bar{\rho}(\mu)(i \mu)^{n} \Phi(i \mu, x, t),
$$

where $d \bar{\rho}$ is the measure constructed for $q(x, 0)$ in the previous section. Then indeed

$$
H_{0}(x, t)=\int_{0}^{\infty} \Phi_{*}(i \mu, x, t) d \bar{\rho}(\mu) \Phi(i \mu, x, t) .
$$

It follows from the uniqueness of the analytic solution $q(x, t)$ of the $\mathrm{KdV}$ equation (1) at the strip $\mathbb{R} \times[0, T]$. The entry $h_{0}^{22}(x, t)$ is chosen from this equality and will satisfy the last equation of (35), which holds for $\int_{0}^{\infty} \Phi_{*}(i \mu, x, t) d \bar{\rho}(\mu) \Phi(i \mu, x, t)$ by the construction.

We can define the following operators:

$$
\begin{aligned}
& B_{*}(x, t)=\frac{1}{2 \pi i} \int_{\Gamma}\left(\lambda I+A_{\zeta}\right)^{-1} B_{0} \Phi_{*}^{*}(\bar{\lambda}, x, t) d \lambda, \\
& C_{*}(x, t)=\frac{1}{2 \pi i} \int_{\Gamma} \Phi_{*}(\lambda, x, t) C_{0}(\lambda I-A)^{-1} d \lambda, \\
& \mathbb{X}_{*}(x, t)=I-\int_{\Gamma} \int_{\Gamma} d \lambda\left(\lambda I+A_{\zeta}\right)^{-1} B_{0} \frac{\Phi_{*}^{*}(\bar{\lambda}, x, t) \sigma_{1} \Phi_{*}(\mu, x, t)-\sigma_{1}}{\lambda-\mu} C_{0}(\mu I-A)^{-1} d \mu,
\end{aligned}
$$

where $\Gamma$ is in the sector of regularity of $A, A_{\zeta}$ as in the previous section. Along with differential equations (46), (47), (48) these operators will also satisfy analogues of (51), (52), (53) as follows:

$$
\begin{array}{cc}
\frac{\partial}{\partial t} B_{*} & =-i A_{\zeta} \frac{\partial}{\partial x} B_{*}-i B_{*} \sigma_{1} \frac{\partial}{\partial x} H_{0}, \\
\frac{\partial}{\partial t} C_{*} u & =i \frac{\partial}{\partial x} C_{*} A u+i \frac{\partial}{\partial x}\left[H_{0}\right] \sigma_{1} C_{*} u \\
\frac{\partial}{\partial t} \mathbb{X}_{*} v=\left(i A_{\zeta} B_{*} \sigma_{2} C_{*}-i B_{*} \sigma_{2} C_{*} A-i B_{*} \gamma_{*} C_{*}\right) v
\end{array}
$$

One can argue that these differential equations actually serve as defining ones for the operators $B_{*}, C_{*}, \mathbb{X}_{*}$ with the initial conditions at $t=0 \mathbb{X}_{*}(x) B(x), C(x) \mathbb{X}_{*}(x), \mathbb{X}_{*}(x)$ defined in the previous section.

Lemma 5.5 (Uniqueness of the moments). Suppose that $H_{n}(x, t)$ and $\widetilde{H}_{n}(x, t)$ are two sequences of moments, which are analytic in $x, t$, satisfy (55) and $H_{n}(x, 0)=\widetilde{H}_{n}(x, 0)$ for all $x \in \mathbb{R}$. Then $H_{n}(x, t)=\widetilde{H}_{n}(x, t)$ for all $x, t$. 
Proof: It is mmediate from (55), because for $n=0$, for example,

$$
\begin{aligned}
& \frac{\partial}{\partial t} H_{0}(x, 0)=i \frac{\partial}{\partial x} H_{1}(x, 0)+i \frac{\partial}{\partial x}\left[H_{0}\right](x, 0) \sigma_{1} H_{0}(x, 0)= \\
&\left.=i \frac{\partial}{\partial x} \widetilde{H}_{1}(x, 0)+i \frac{\partial}{\partial x} \widetilde{[} H_{0}\right](x, 0) \sigma_{1} \widetilde{H}_{0}(x, 0)=\frac{\partial}{\partial t} \widetilde{H}_{0}(x, 0) .
\end{aligned}
$$

Continuing in the same manner by induction, we will obtain that

$$
\frac{\partial}{\partial t} H_{0}^{(n)}(x, 0)=\frac{\partial}{\partial t} \widetilde{H}_{0}^{(n)}(x, 0)
$$

and the Lemma follows.

Corollary 5.6. the following equality holds

$$
C_{*}(x, t) A^{n} B(x, t)=H_{n}(x, t) .
$$

Proof: Since $C_{*}(x, 0) A^{n} B(x, 0)=H_{n}(x, 0)$ and differentiating

$$
\begin{aligned}
\frac{\partial}{\partial t}\left[C_{*} A^{n} B\right]=i \frac{\partial}{\partial x} C_{*} A^{n+1} B++i \frac{\partial}{\partial x}\left[H_{0}\right] \sigma_{1} C_{*} A^{n} B+ & C^{*} A^{n} i A B_{x}= \\
& =i \frac{\partial}{\partial x}\left[C_{*} A^{n+1} B\right]+i \frac{\partial}{\partial x}\left[H_{0}\right] \sigma_{1} C_{*} A^{n} B,
\end{aligned}
$$

we obtain that $C_{*} A^{n} B, H_{n}$ satsify the same differential equations with identical initial conditions, so they are equal by Lemma 5.5. Particularly, for $n=0$ we obtain that $C_{*}(x, t) B(x, t)=H_{0}(x, t)$.

From this Lemma it follows that Theorem 4.10 holds, using the same idea of proof, but with the $t$-derivatives

Theorem 5.7. The following equalities hold:

$$
\begin{gathered}
\mathbb{X}(x, t) B_{*}(x, t)=B(x, t), \quad C_{*}(x, t) \mathbb{X}(x, t)=C(x, t), \\
\mathbb{X}_{*}(x, t) B(x, t)=B_{*}(x, t), \quad C(x, t) \mathbb{X}_{*}(x, t)=C_{*}(x, t) .
\end{gathered}
$$

Proof: We will mimic the proof of Theorem 4.10.

$$
\begin{aligned}
\frac{\partial}{\partial t}\left[C_{*} \mathbb{X}\right] & =i \frac{\partial}{\partial x} C_{*} A \mathbb{X}+i \frac{\partial}{\partial x}\left[H_{0}\right] \sigma_{1} C_{*} \mathbb{X}+C_{*}\left[i A B \sigma_{2} C-i B \sigma_{2} C A_{\zeta}+i B \gamma C\right] \\
& =\text { using (11), (61), (47) } \\
& =-i \frac{\partial}{\partial x}\left[C_{*} \mathbb{X}\right] A_{\zeta}+i \frac{\partial}{\partial x}\left[H_{0}\right] \sigma_{1}\left[C_{*} \mathbb{X}-C\right] .
\end{aligned}
$$

Plugging here $C$ instead of $C_{*} \mathbb{X}$, we will obtain (52) for $C$ and by the uniqueness of the solutions since for $t=0$ it holds that $C_{*}(x, 0) \mathbb{X}(x, 0)=C(x, 0)$ (Theorem 4.10), we obtain that also $C_{*}(x, t) \mathbb{X}(x, t)=$ $C(x, t)$.

In a similar manner one can obtain that

$$
\frac{\partial}{\partial t}\left[\mathbb{X}_{*} B\right]=-i A_{\zeta} \frac{\partial}{\partial x}\left[\mathbb{X}_{*} B\right]-i B_{*} \sigma_{1} \frac{\partial}{\partial x}\left[H_{0}\right]
$$

Since $B_{*}$ when substituted here instead of $\mathbb{X}_{*} B$ satisfies the same equation, and $\mathbb{X}_{*}(x, 0) B(x, 0)=$ $B_{*}(x, 0)$ (Theorem 4.10), we obtain that $\mathbb{X}_{*}(x, t) B(x, t)=B_{*}(x, t)$. Then as before

$$
C(x, t) B_{*}(x, t)=C(x, t) \mathbb{X}_{*}(x, t) B(x, t)=C_{*}(x, t) B(x, t)=H_{0}(x, t) .
$$

The equations $\mathbb{X}(x, t) B_{*}(x, t)=B(x, t), \quad C(x, t) \mathbb{X}_{*}(x, t)=C_{*}(x, t)$ follow in the same manner. 
Corollary 5.8. The operator $\mathbb{X}(x, t)$ is invertible for all $x \in \mathbb{R}, t \in[0, T]$ with the inverse $\mathbb{X}_{*}(x, t)$.

Proof: As in Corollary 4.11 we obtain that

$$
\frac{\partial}{\partial x}\left[\mathbb{X}(x, t) \mathbb{X}_{*}(x, t)\right]=\frac{\partial}{\partial x}\left[\mathbb{X}_{*}(x, t) \mathbb{X}(x, t)\right]=0 .
$$

Differentiating with respect to $t$, we obtain that

$$
\begin{aligned}
& \frac{\partial}{\partial t}\left[\mathbb{X}(x, t) \mathbb{X}_{*}(x, t)\right]=(153),(63) \\
& =\left(i A B \sigma_{2} C-i B \sigma_{2} C A_{\zeta}+i B \gamma C\right) \mathbb{X}_{*}+\mathbb{X}\left(i A_{\zeta} B_{*} \sigma_{2} C_{*}-i B_{*} \sigma_{2} C_{*} A-i B_{*} \gamma_{*} C_{*}\right) \\
& =\text { Theorem [5.7 } \\
& =i A B \sigma_{2} C_{*}-i B \sigma_{2} C A_{\zeta} \mathbb{X}_{*}+i B \gamma C_{*}+i \mathbb{X} A_{\zeta} B_{*} \sigma_{2} C_{*}-i B \sigma_{2} C_{*} A-i B \gamma_{*} C_{*} \\
& =(6),(48) \\
& =i A B \sigma_{2} C_{*}-i B \sigma_{2} C\left[-\mathbb{X}_{*} A-B_{*} \sigma_{1} C_{*}\right]+i B \gamma C_{*}+i\left[-A \mathbb{X}-B \sigma_{1} C\right] B_{*} \sigma_{2} C_{*}-i B \sigma_{2} C_{*} A-i B \gamma_{*} C_{*} \\
& =i B \sigma_{2} C B_{*} \sigma_{1} C_{*}+i B \gamma C_{*}-i B \sigma_{1} C B_{*} \sigma_{2} C_{*}-i B \gamma_{*} C_{*} \\
& =i B\left[\sigma_{2} H_{0} \sigma_{1}+\gamma-\sigma_{1} H_{0} \sigma_{2}-\gamma_{*}\right] C_{*}= \\
& =0
\end{aligned}
$$

Similarly, one shows that $\frac{\partial}{\partial t}\left[\mathbb{X}_{*}(x, t) \mathbb{X}(x, t)\right]=0$. Thus the operator $\mathbb{X}(x, t) \mathbb{X}_{*}(x, t)$ is analytic and has zero derivatives with respect to $t$, and $x$. Thus it is constant. Since at $x=t=0$ it is identity, the result follows.

Theorem 5.9. Suppose that $q(x, t)$ is a solution of (1) on $\mathrm{R} \times[0, T]$, then there exists a KdV vessel, realizing $q(x, t)$ on $\Omega$, so that $\mathrm{R} \times[0, T] \subseteq \Omega$.

Proof: For $q(x, 0)$ we construct the SL vessel $\mathfrak{V}$. Since the solution $q(x, t)$ exists on $\mathrm{R} \times[0, T]$ the fundamental matrix $\Phi_{*}(\lambda, x, t)$ of (19) exists for all $(x, t) \in \mathrm{R} \times[0, T]$. We can also define the ingredients of the "inverse vessel" $B_{*}(x, t), \mathbb{X}_{*}(x, t), C_{*}(x, t)$ by formulas 600 . Then by Corollary $5.8 \mathbb{X}_{*}(x, t)$ is the inverse of $\mathbb{X}(x, t)$ on $\mathbb{R} \times[0, T)$. So, $\Omega$ includes the set $\mathrm{R} \times[0, T]$.

\section{Remarks}

1. A next step, related to this research, would be a development of a similar theory for locally integrable functions $q(x)$ on $\mathbb{R}$. Using approximations by analytic functions similar results should be obtainable.

2. In the case $q(x)$ is analytic and satisfies $\int_{-\infty}^{\infty}(1+|x|) q(x) d x<\infty$ [Fad74] one obtains that the fundamental matrices $\Phi_{*}(i \mu, x), \Phi_{*}\left(-A_{\zeta}, x\right)$ are UNIFORMLY bounded, and as a result the operator $\mathbb{Y}^{*}(x)$ is uniformly bounded as well. Thus there exists $T=T_{x}$ - the same for all $x$, so that the operator $\mathbb{X}(x, t)$ is invertible. It follows that there exists a local solution on $\mathbb{R} \times[-T, T]$ of the $\mathrm{KdV}$ equation (1), which is a very well known result for the KdV equation GGKM67, Fad74.

3. These vessel constructions are intimately related to the theory of systems Sta05. Their relations and interplay is left for a future work.

\section{References}

[AMV] D. Alpay, A. Melnikov, and V. Vinnikov. On the class SI of conservative functions intertwining solutions of linear differential equations. http://arxiv.org/abs/0912.2014. 
[AMV12] D. Alpay, A. Melnikov, and V. Vinnikov. Schur algorithm in the class $I$ of $J$-contractive functions intertwining solutions of linear differential equations. IEOT, 74(3):313-344, 2012.

[BGR90] J. Ball, I. Gohberg, and L. Rodman. Interpolation of rational matrix functions. Operator Theory: Advances and Applications. Birkhäuser Verlag, Basel, 1990.

[BJ02] J. Bourgain and S. Jitomirskaya. Absolutely continuous spectrum for 1D quasi-periodic operators. Invent. Math., 148:453-463, 2002.

[BL58] M.S. Brodskii and M.S. Livšic. Spectral analysis of non-self-adjoint operators and intermediate systems (Russian). Uspehi Mat. Nauk (N.S.), 13(1 (79)):3-85, 1958.

[Boa39] Jr. Boas, R. P. The Stieltjes moment problem for functions of bounded variation. Bull. Amer. Math. Soc., 45(6):399-404, 1939.

[Bro71] M.S. Brodskii. Triangular and Jordan representations of linear operators. Translations of AMS, 1971.

[CL55] E.A Coddington and N. Levinson. Theory of ordinary differential equation. Mc-Graw Hill, 1955.

[Cru55] M.M. Crum. Associated Sturm-Liouville systems. Quart. J. Math. Oxford Ser., 6(2):121-127, 1955.

[DLdS] A. Diksma, H. Langer, and H.S.V. de Snoo. Representations of holomorphic operator functions by means of resolvents of unitary or self-adjoint operators in Krein spaces. Operator Theory: Adv. and App., 24:123-143. Birkhauser Verlag, Berlin.

[DS75] E.I. Dinaburg and Y. G. Sinai. The one dimensional Schrödinger equation with quasiperiodic potential. Funkt. Anal. i. Priloz., 9:8-21, 1975.

[DZ91] P. Deift and X. Zho. Direct and inverse scattering on the line with arbitrary singularities. Comm. on Pure and Appl. math., 44(5):485-533, 1991.

[EN00] Klaus-Jochen Engel and Rainer Nagel. One-parameter semigroups for linear evolution equations. Springer, 2000.

[Fad74] L.D. Fadeev. The inverse problem in the quantum theory of scattering, II. Itogi Nauk. i Techn., 4:93-180, 1974.

[GD] M. Goldstein and D. Damanik. On the Existence and Uniqueness of Global Solutions for the KdV Equation with Quasi-Periodic Initial Data. http://arxiv.org/abs/1212.2674.

[GGKM67] C.S. Gardner, J.M. Greene, M.D. Kruskal, and R.M. Miura. Method for solving the Korteweg-de Vries equation. Phys. Rev. Lett., 19:1095-1097, 1967.

[GK69] I. Gohberg and M. Krein. Introduction to the theory of linear non-selfadjoint operators. translations of AMS, 1969.

[GL51] I. M. Gelfand and B.M. Levitan. On the determination of a differential equation from its spectral function (Russian). Izvestiya Akad. Nauk SSSR. Ser. Mat., 15, 1951.

[KdV95] D. J. Korteweg and G. de Vries. On the change of form of long waves advancing in a rectangular canal, and on a new type of long stationary waves. Philosophical Magazine, 39:422-443, 1895.

[Kri77] I.M. Krichever. Methods of algebraic geometry in a theory of nonlinear equations. Usp. Math. Nauk, 32(6):183-208, 1977.

[LS75] B. M. Levitan and I. S Sargsjan. Introduction to spectral theory: selfadjoint ordinary differential operators, volume 39. Translations of Mathematical Monographs, Providence, R.I., 1975. 
[Ls01] M.S. Livšic. Vortices of 2D systems. Operator Theory: Advances and Applications, 123:7-41, 2001.

[Mar77] V. A. Marčenko. Sturm Liouville operators and their applications. Naukova Dumka, Kiev, 1977.

[Mela] A. Melnikov. Construction of a Sturm-Liouville vessel using Gelfand-Levitan theory. On solution of the Korteweg-de Vries equation in the first quadrant. http://arxiv.org/abs/1212.1730.

[Melb] A. Melnikov. On a theory of vessels and the inverse scattering. http://arxiv.org/abs/1103.2392.

[Melc] A. Melnikov. On completely integrable polynomial PDEs arising from Sturm-Liouville differential equation using evolutionary vessels. KdV hierarchy. http://arxiv.org/abs/1206.2909.

[Meld] A. Melnikov. On construction of solutions of the evolutionary Non Linear Schrödinger equation. http://arxiv.org/abs/1209.0179.

[Mele] A. Melnikov. Solution of the Boussinesq equation using evolutionary vessels. http://arxiv.org/abs/1301.2573.

[Melf] A. Melnikov. Solution of the KdV equation using evolutionary vessels. http://arxiv.org/abs/1110.3495.

[Mel11] A. Melnikov. Finite dimensional Sturm Liouville vessels and their tau functions. IEOT, 71(4):455-490, 2011.

[MW66] W. Magnus and S. Winkler. Hill's equation, volume Interscience Tracts in Pure and Applied Mathematics, Interscience Publishers John Wiley and Sons. Naukova Dumka, New York-London-Sydney", 1966.

[Sta05] O. Staffans. Well-Posed linear systems. Encyclopedia of math. Cambridge, 2005. 